\title{
New results on noncompact harmonic manifolds
}

\author{
Gerhard Knieper
}

\begin{abstract}
The Lichnerowicz conjecture asserts that all harmonic manifolds are either flat or locally symmetric spaces of rank 1. This conjecture has been proved by Z. I. Szabó [Sz] for harmonic manifolds with compact universal cover. E. Damek and F. Ricci [DR] provided examples showing that in the noncompact case the conjecture is wrong. However, such manifolds do not admit a compact quotient.

In this paper we study, using a notion of rank, the asymptotic geometry and the geodesic flow on simply connected nonflat and noncompact harmonic manifolds denoted by $X$.

In the first part of the paper we show that the following assertions are equivalent. The volume growth is purely exponential, the rank of $X$ is one, the geodesic flow is Anosov with respect to the Sasaki metric, $X$ is Gromov hyperbolic. We also provide a characterization of manifolds of constant negative curvature by their minimal volume growth among those harmonic manifolds with fixed mean curvature of the horospheres.

In the second part of the paper we show that the geodesic flow is Anosov if $X$ is a nonflat harmonic manifold with no focal points. In the course of the proof we obtain that certain partially hyperbolic flows on arbitrary Riemannian manifolds without focal points are Anosov, which is of interest beyond harmonic manifolds.

Combining the results of this paper with the rigidity theorem's of [BCG], [BFL], and [FL], we confirm the Lichnerowicz conjecture for all compact harmonic manifolds without focal points or with Gromov hyperbolic fundamental groups.
\end{abstract}

Mathematics Subject Classification (2010). Primary 37C40; Secondary 53C12, 37C10.

Keywords. Harmonic manifolds, geodesic flows, Lichnerowicz conjecture.

\section{Introduction}

A complete Riemannian manifold $X$ is called harmonic if the harmonic functions satisfy the mean value property, that is, the average on any sphere coincides with its value in the center. Equivalently, for any $p \in X$ the volume density $\theta_{p}(q)=$ $\sqrt{\operatorname{det} g_{i j}(q)}$ in normal coordinates, centered at any point $p \in X$, is a radial function. In particular, if $c:[0, \infty) \rightarrow X$ is a normal geodesic with $c(0)=p$, the function $f(t):=\theta_{p}(c(t))$ is independent of $c$. It is easy to see that all rank 1 symmetric spaces and Euclidean spaces (model spaces) are harmonic. In 1944, A. Lichnerowicz conjectured that conversely every complete harmonic manifold is a model space. He confirmed the conjecture up to dimension 4 [Li]. It was not before the beginning 
of the 1990s that general results where obtained. In 1990 Z.I. Szabó [Sz] proved the Lichnerowicz conjecture for compact simply connected spaces. However, not much later, in 1992, E. Damek and F. Ricci [DR] showed that in the noncompact case the conjecture is wrong. They provided examples of homogeneous harmonic spaces which are not symmetric. Nevertheless, in 1995 G. Besson, G. Courtois and S. Gallot $[\mathrm{BCG}]$ confirmed the conjecture for manifolds of negative curvature admitting a compact quotient. The proof consisted in a combination of deep rigidity results from hyperbolic dynamics and used besides [BCG] the work of Y. Benoist, P. Foulon and F. Labourie ([BFL] and P. Foulon and F. Labourie [FL]).

In 2002, A. Ranjan and H. Shah showed [RSh2] that noncompact, simply connected harmonic manifolds of polynomial volume growth are flat. Using a result by Y. Nikolayevski [Ni] showing that the density function $f$ is an exponential polynomial, subexponential volume growth of noncompact simply connected harmonic manifolds implies flatness as well. In 2006, J. Heber [He] proved that among the homogeneous harmonic spaces only the model spaces and the Damek-Ricci spaces occur. Therefore, it remains to study nonhomogeneous harmonic manifolds of exponential volume growth. In particular, these are spaces without conjugate points and horospheres of constant mean curvature $h>0$.

The starting point of this paper was a question raised by N. Peyerimhoff whether noncompact simply connected harmonic manifolds, whose horospheres have positive mean curvature $h>0$ have purely exponential volume growth, i.e., the quotient of the density function $f(t)$ and $e^{h t}$ stays for large $t$ between two positive constants.

It turned out that the answer to this question is intimately related to the notion of rank, which is a straight forward generalization of the well-known rank of manifolds of nonpositive curvature [BBE]. In particular, the volume growth is purely exponential if and only if the rank is 1 . Moreover, we show that noncompact harmonic manifolds are of rank 1 if and only if the geodesic flow is Anosov. Therefore, having a compact quotient, the rigidity theorems mentioned above force harmonic spaces of rank 1 as in the case of negative curvature to be locally symmetric.

We believe that all nonflat harmonic manifolds are of rank 1 . This would imply the Lichnerowicz conjecture for all compact manifolds which is one of the ultimate goals in the future investigations. We confirm the rank 1 condition for instance for all harmonic manifolds without focal points which include spaces of nonpositive curvature. It is very likely that all noncompact harmonic manifolds have no focal points. All known examples of noncompact harmonic manifolds have nonpositive curvature. In [RSh1] it was shown that a sufficient condition for no focal points is provided under the assumption that besides the trace also the determinant of the second fundamental form of the geodesic spheres is a function of the radius.

The paper is organized as follows. In Section 2 we introduce the techniques of Jacobi tensors. In particular, we show that manifolds of constant negative curvature have minimal volume growth among those harmonic manifolds with fixed mean curvature of the horospheres. 
In Section 3 we introduce the notion of rank for manifolds without conjugate points. We show that for harmonic spaces the following three properties are equivalent: $X$ has rank 1, the geodesic flow on $X$ is Anosov, the volume growth is purely exponential.

In Section 4 we show that for noncompact simply connected harmonic spaces, Gromov hyperbolicity is equivalent to the three properties studied in Section 3. Hence, compact harmonic spaces with Gromov hyperbolic fundamental groups are locally symmetric.

In Section 5 we study harmonic manifolds $X$ of bounded asymptote and show that the rank is constant. In particular, they include all manifolds without focal points, i.e., manifolds for which geodesic spheres are convex. Using a classical topological result of N. E. Steenrod and J. H. C. Whitehead on vector fields of spheres [SW], we show that in odd dimensions the rank is one. If additionally $X$ admits a compact quotient, $X$ has to have constant negative curvature.

In Section 6 we study geodesic flows on arbitrary manifolds without focal points and constant rank. Under the assumption of bounded sectional curvature together with a certain transversality condition, we prove first that the geodesic flow is partially hyperbolic. Using a geometric argument we finally show that the flow is Anosov. By adding to this the results of chapter 5, we obtain that for all harmonic manifolds without focal points the geodesic flow is Anosov as well.

In the appendix we collect for the convenience of the reader properties of Jacobi tensors which are important in this paper.

\section{Volume growth in harmonic manifolds}

In this paper $X$ be will denote a complete, noncompact, simply connected harmonic manifold. This implies that $X$ is a manifold without conjugate points and thus, by a theorem of Cartan-Hadamard, the exponential map $\exp _{p}: T_{p} X \rightarrow X$ is a diffeomorphism. Moreover, $X$ is an Einstein manifold and thus analytic (see [Be]).

We briefly recall the calculus of Jacobi tensors (see e.g. [Es], [Gre], [Kn2] and [Kn] for more details). Let $c: I \rightarrow X$ be a unit speed geodesic and let $N(c)$ denote the normal bundle of $c$ given by a disjoint union

$$
N_{t}(c):=\left\{w \in T_{c(t)} X \mid\langle w, \dot{c}(t)\rangle=0\right\} .
$$

A $(1,1)$-tensor along $c$ is a differentiable section

$$
Y: \mathbb{R} \rightarrow \text { End } N(c)=\bigcup_{t \in I} \operatorname{End}\left(N_{t}(c)\right),
$$

i.e., for all orthogonal parallel vector fields $x_{t}$ along $c$ the covariant derivative of 
$t \rightarrow Y(t) x_{t}$ exists. The derivative $Y^{\prime}(t) \in \operatorname{End}\left(N_{t}(c)\right)$ is defined by

$$
Y^{\prime}(t)\left(x_{t}\right)=\frac{D}{d t}\left(Y(t) x_{t}\right) .
$$

$Y$ is called parallel if $Y^{\prime}(t)=0$ for all $t$. If $Y$ is parallel we have $Y(t) x_{t}=(Y(0) x)_{t}$ and, therefore, $\left\langle Y(t) x_{t}, y_{t}\right\rangle$ is constant for all parallel vector fields $x_{t}, y_{t}$ along $c$. In particular, $Y$ is parallel if and only if $Y$ is a constant matrix with respect to parallel frame field in the normal bundle of $c$. Therefore, parallel $(1,1)$-tensors are also called constant.

The curvature tensor $R$ induces a symmetric $(1,1)$-tensor along $c$ given by

$$
R(t) w:=R(w, \dot{c}(t)) \dot{c}(t) .
$$

A $(1,1)$-tensor $Y$ along $c$ is called a Jacobi tensor if it solves the Jacobi equation

$$
Y^{\prime \prime}(t)+R(t) Y(t)=0 .
$$

If $Y, Z$ are two Jacobi tensors along $c$ the derivative of the Wronskian

$$
W(Y, Z)(t):=Y^{\prime *}(t) Z(t)-Y^{*}(t) Z^{\prime}(t)
$$

is zero and thus, $W(Y, Z)$ defines a parallel $(1,1)$-tensor. A Jacobi tensor $Y$ along a geodesic $c: I \rightarrow X$ is called Lagrange tensor if $W(Y, Y)=0$. The importance of Lagrange tensors comes from the following proposition.

Proposition 2.1. Let $Y: I \rightarrow$ End $N(c)$ be a Lagrange tensor along a geodesic $c: I \rightarrow X$ which is nonsingular for all $t \in I$. Then for $t_{0} \in I$ and any other Jacobi tensor $Z$ along $c$, there exist constant tensors $C_{1}$ and $C_{2}$ such that

$$
Z(t)=Y(t)\left(\int_{t_{0}}^{t}\left(Y^{*} Y\right)^{-1}(s) d s C_{1}+C_{2}\right)
$$

for all $t \in I$.

Remark. The definition of the integral and a proof of this proposition is given in the appendix.

Let $S X$ denote the unit tangent bundle of $X$ with fibres $S_{p} X, p \in X$, and, for every $v \in S X$, let $c_{v}: \mathbb{R} \rightarrow X$ denote the unique geodesic satisfying $\dot{c}_{v}(0)=v$. Define $A_{v}$ to be the Jacobi tensor along $c_{v}$ with $A_{v}(0)=0$ and $A_{v}^{\prime}(0)=$ id. Then the volume of a geodesic sphere $S(p, r)$ of radius $r$ about $p$ is given by

$$
\operatorname{vol} S(p, r)=\int_{S_{p} X} \operatorname{det} A_{v}(r) d \theta_{p}(v),
$$


where $d \theta_{p}(v)$ is the volume element of $S_{p} X$ induced by the Riemannian metric. By definition $X$ is harmonic if and only if the volume density $f(t)=\operatorname{det} A_{v}(t)$ does not depend on $v$. Therefore,

$$
\operatorname{vol} S(p, r)=\omega_{n-1} f(r),
$$

where $\omega_{n-1}$ is the volume of the sphere in the Euclidean space $\mathbb{R}^{n}$. Since

$$
\frac{\left(\operatorname{det} A_{v}(r)\right)^{\prime}}{\operatorname{det} A_{v}(r)}=\operatorname{tr}\left(A_{v}^{\prime}(r) A_{v}(r)^{-1}\right)
$$

is the mean curvature of the geodesic sphere of radius $r>0$ about $\pi(v)$ in $c_{v}(r), X$ is harmonic if and only if the mean curvature of all spheres is a function depending only on the radius.

Of fundamental importance are the stable and unstable Jacobi tensors. For a general complete simply connected manifold without conjugate points $X$ they are defined as follows. For $v \in S X$ and $r>0$ denote by $S_{v, r}$ and $U_{v, r}$ the Jacobi tensors along $c_{v}$ such that

$$
S_{v, r}(0)=U_{v, r}(0)=\mathrm{id} \quad \text { and } \quad S_{v, r}(r)=0, U_{v, r}(-r)=0 .
$$

Let

$$
S_{v}=\lim _{r \rightarrow \infty} S_{v, r} \quad \text { and } \quad U_{v}=\lim _{r \rightarrow \infty} U_{v, r}
$$

be the stable and unstable Jacobi tensors. For each $r>0$ we have

$$
W\left(S_{v, r}, S_{v, r}\right)(0)=S_{v, r}^{\prime *}(0)-S_{v, r}^{\prime}(0)=W\left(S_{v, r}, S_{v, r}\right)(r)=0
$$

and

$$
W\left(U_{v, r}, U_{v, r}\right)(0)=U_{v, r}^{\prime *}(0)-U_{v, r}^{\prime}(0)=W\left(U_{v, r}, U_{v, r}\right)(-r)=0,
$$

which implies that the Jacobi tensors $S_{v, r}$ and $U_{v, r}$ are Lagrangian and the endomorphisms $S_{v, r}^{\prime}(0)$ and $U_{v, r}^{\prime}(0)$ are symmetric. By passing to the limit, the stable and unstable Jacobi tensors $S_{v}$ and $U_{v}$ are Lagrangian and the endomorphisms $S_{v}^{\prime}(0)$ and $U_{v}^{\prime}(0)$ are symmetric as well. Note, that $\operatorname{tr} U_{v, r}^{\prime}(0)=\operatorname{tr}\left(A_{\phi^{-r} v}^{\prime}(r) A_{\phi^{-} r_{v}}^{-1}(r)\right)$ and if $X$ is a complete noncompact harmonic manifold tr $U_{v, r}^{\prime}(0)=\frac{f^{\prime}(r)}{f(r)}$ is converging to $\operatorname{tr} U_{v}^{\prime}(0)=: h \geq 0$, where $h$ is the mean curvature of the horospheres. Hence,

$$
\lim _{r \rightarrow \infty} \frac{\log \operatorname{vol} S(p, r)}{r}=\lim _{r \rightarrow \infty} \frac{f^{\prime}(r)}{f(r)}=h .
$$

Definition 2.2. A noncompact simply connected harmonic manifold with $h>0$ is called of purely exponential volume growth if there are constants $0<a \leq b$ such that

$$
a e^{h r} \leq f(r) \leq b e^{h r}
$$

for all $r \geq 1$. 
Remark. The notion of purely exponential volume growth has been introduced in [Kn1]. In particular, in [Kn1] it was proved that simply connected manifolds of nonpositive curvature and geometric rank 1 have purely exponential volume growth provided they admit a compact quotient.

Lemma 2.3. Let $X$ be a complete simply connected manifold without conjugate points. Let $c_{v}: \mathbb{R} \rightarrow X$ be a geodesic with $\dot{c}_{v}(0)=v \in S X$ and $s, r>0$. Then we have

$$
\left(U_{v, r}^{\prime}(0)-S_{v, s}^{\prime}(0)\right)^{-1}=\int_{0}^{s}\left(U_{v, r}^{*} U_{v, r}\right)^{-1}(u) d u
$$

and

$$
\left(U_{v}^{\prime}(0)-S_{v, s}^{\prime}(0)\right)^{-1}=\int_{0}^{s}\left(U_{v}^{*} U_{v}\right)^{-1}(u) d u .
$$

Similarly, for $0<s<r$ we have

$$
\left(S_{v, r}^{\prime}(0)-S_{v, s}^{\prime}(0)\right)^{-1}=\int_{0}^{s}\left(S_{v, r}^{*} S_{v, r}\right)^{-1}(u) d u
$$

and

$$
\left(S_{v}^{\prime}(0)-S_{v, s}^{\prime}(0)\right)^{-1}=\int_{0}^{s}\left(S_{v}^{*} S_{v}\right)^{-1}(u) d u .
$$

Furthermore, the function

$$
\operatorname{det}\left(\int_{0}^{s}\left(U_{v}^{*} U_{v}\right)^{-1}(u) d u\right)=\frac{1}{\operatorname{det}\left(U_{v}^{\prime}(0)-S_{v, s}^{\prime}(0)\right)}
$$

is strictly monotonically increasing.

Proof. Let $s, r$ be positive real numbers. For all $s>-r$ the endomorphism $U_{v, r}(s)$ is nonsingular and Lagrangian. Using Proposition 2.1, for all $t>-r$ we obtain

$$
S_{v, s}(t)=U_{v, r}(t) \int_{t}^{s}\left(U_{v, r}^{*} U_{v, r}\right)^{-1}(u) d u C_{r, s}
$$

for a constant $(1,1)$-tensor $C_{r, s}$. Evaluating and differentiating this identity at $t=0$ yields

$$
\mathrm{id}=S_{v, s}(0)=\int_{0}^{s}\left(U_{v, r}^{*} U_{v, r}\right)^{-1}(u) d u C_{r, s}
$$


and

$$
\begin{aligned}
S_{v, s}^{\prime}(0) & =U_{v, r}^{\prime}(0) \int_{0}^{s}\left(U_{v, r}^{*} U_{v, r}\right)^{-1}(u) d u C_{r, s}-C_{r, s} \\
& =U_{v, r}^{\prime}(0)-C_{r, s}
\end{aligned}
$$

which proves the first equation. Taking on both sides the limit $r \rightarrow \infty$ yields the second equation.

Now consider $0<s<r$. Again using Proposition 2.1, for all $t<r$ we obtain

$$
S_{v, s}(t)=S_{v, r}(t) \int_{t}^{s}\left(S_{v, r}^{*} S_{v, r}\right)^{-1}(u) d u D_{r, s}
$$

for a constant $(1,1)$-tensor $D_{r, s}$. As above evaluating and differentiating this identity at $t=0$ yields the second assertion. Since

$$
0<\left\langle\left(U_{v}^{\prime}(0)-S_{v, s_{2}}^{\prime}(0)\right) x, x\right\rangle<\left\langle\left(U_{v}^{\prime}(0)-S_{v, s_{1}}^{\prime}(0)\right) x, x\right\rangle
$$

for $s_{1}<s_{2}$ and $x \in v^{\perp}$, we obtain the last claim.

Proposition 2.4. Let $X$ be a noncompact, simply connected harmonic manifold and $h=0$. Then $X$ is flat.

Proof. From [Ni] follows that $X$ has polynomial volume growth. But this implies the flatness of $X$ as was shown in [RSh2].

Corollary 2.5. Let $X$ be a noncompact, simply connected harmonic manifold such that $h>0$. Then the function $F:[0, \infty) \rightarrow[0, \infty)$ given by

$$
F(t)=\frac{f(t)}{e^{h t}}=\frac{1}{\operatorname{det}\left(U_{v}^{\prime}(0)-S_{v, t}^{\prime}(0)\right)}
$$

is strictly monotonically increasing. Moreover,

$$
\lim _{t \rightarrow \infty} F(t)= \begin{cases}\infty & \text { if } \operatorname{det}\left(U_{v}^{\prime}(0)-S_{v}^{\prime}(0)\right)=0, \\ \frac{1}{\operatorname{det}\left(U_{v}^{\prime}(0)-S_{v}^{\prime}(0)\right)} & \text { if } \operatorname{det}\left(U_{v}^{\prime}(0)-S_{v}^{\prime}(0)\right)>0 .\end{cases}
$$

In particular, $\operatorname{det}\left(U_{v}^{\prime}(0)-S_{v}^{\prime}(0)\right)$ and $\operatorname{det}\left(U_{v}^{\prime}(0)-S_{v, t}^{\prime}(0)\right)$ are independent of $v \in S X$ and the estimate

$$
\frac{e^{h t}}{\operatorname{det}\left(U_{v}^{\prime}(0)-S_{v, 1}^{\prime}(0)\right)} \leq f(t)
$$

holds for all $t \geq 1$. 
Proof. Let $A_{v}$ be the Jacobi tensor along the geodesic $c_{v}: \mathbb{R} \rightarrow X$ with $\dot{c}_{v}(0)=v \in$ $S X$ such that $A_{v}(0)=0$ and $A_{v}^{\prime}(0)=$ id. Then Proposition 2.1 implies

$$
A_{v}(t)=U_{v}(t) \int_{0}^{t}\left(U_{v}^{*} U_{v}\right)^{-1}(u) d u .
$$

Since by Lemma 7.3 we have

$$
\left(\log \operatorname{det} U_{v}\right)^{\prime}(t)=\operatorname{tr} U_{v}^{\prime}(t) U_{v}^{-1}(t)=\operatorname{tr} U_{\phi^{t}(v)}^{\prime}(0)=h,
$$

we obtain

$$
\begin{aligned}
\frac{f(t)}{e^{h t}} & =\frac{\operatorname{det} A_{v}(t)}{\operatorname{det} U_{v}(t)}=\operatorname{det}\left(\int_{0}^{t}\left(U_{v}^{*} U_{v}\right)^{-1}(u) d u\right) \\
& =\frac{1}{\operatorname{det}\left(U_{v}^{\prime}(0)-S_{v, t}^{\prime}(0)\right)} .
\end{aligned}
$$

In particular, $\operatorname{det}\left(U_{v}^{\prime}(0)-S_{v, t}^{\prime}(0)\right)$ as well as $\operatorname{det}\left(U_{v}^{\prime}(0)-S_{v}^{\prime}(0)\right)$ are independent of $v \in S X$. The stated estimate follows from the fact that $\operatorname{det}\left(U_{v}^{\prime}(0)-S_{v, t}^{\prime}(0)\right)$ is monotonically decreasing in $t$.

Using the result above we obtain that manifolds of constant negative curvature have minimal volume growth among those harmonic manifolds with fixed mean curvature of the horospheres. More precisely:

Corollary 2.6. Let $X$ be an n-dimensional, noncompact, simply connected harmonic manifold with mean curvature of the horospheres equal to $h>0$. Then

$$
\lim _{t \rightarrow \infty} \frac{f(t)}{e^{h t}} \geq\left(\frac{n-1}{2 h}\right)^{n-1}
$$

and equality holds if and only if $X$ has constant negative sectional curvature.

Proof. Note, that for a given symmetric matrix $B$ on $\mathbb{R}^{k}$ with positive eigenvalues, we have $(\operatorname{det} B)^{1 / k} \leq \frac{\operatorname{tr} B}{k}$, where equality holds if and only if $B=\lambda$ id. Applying this to $B=\left(U_{v}^{\prime}(0)-S_{v}^{\prime}(0)\right)$, we obtain from the theorem above that

$$
\lim _{t \rightarrow \infty} \frac{f(t)}{e^{h t}} \geq\left(\frac{n-1}{2 h}\right)^{n-1},
$$

where equality holds if and only if $\left(U_{v}^{\prime}(0)-S_{v}^{\prime}(0)\right)=\frac{2 h}{n-1}$ id. Let us assume that equality holds. Consider $U(v)=U_{v}^{\prime}(0)$ and $S(v)=S_{v}^{\prime}(0)$ then they are both solutions of the Riccati equation (see e.g. [Gre]), i.e.

$$
\frac{d}{d t} U\left(\phi^{t}(v)\right)+U^{2}\left(\phi^{t}(v)\right)+R_{v}(t)=0
$$


and

$$
\frac{d}{d t} S\left(\phi^{t}(v)\right)+S^{2}\left(\phi^{t}(v)\right)+R_{v}(t)=0,
$$

where $\phi^{t}: S X \rightarrow S X$ denotes the geodesic flow on the unit tangent bundle $S X$ and $R_{v}(t)$ is the Jacobi operator induced by the curvature tensor $R$, i.e., $R_{v}(t)(x)=$ $R\left(x, \phi^{t}(v)\right) \phi^{t}(v)$ for $x \in \phi^{t}(v)^{\perp}$. Subtracting the two Riccati equations, we obtain

$$
0=\left.\frac{d}{d t}\right|_{t=0} U\left(\phi^{t}(v)\right)-\left.\frac{d}{d t}\right|_{t=0} S\left(\phi^{t}(v)\right)+U^{2}(v)-S^{2}(v)=U^{2}(v)-S^{2}(v),
$$

and hence

$$
U^{2}(v)=\left(\frac{2 h}{n-1} \mathrm{id}+S(v)\right)^{2}=\left(\frac{2 h}{n-1}\right)^{2} \mathrm{id}+\frac{4 h}{n-1} S(v)+S(v)^{2} .
$$

Since $S^{2}(v)=U^{2}(v)$, this implies

$$
S(v)=-\frac{h}{n-1} \mathrm{id}
$$

and, therefore, using the Riccati equation again we obtain

$$
R_{v}(0)=-S(v)^{2}=-\left(\frac{h}{n-1}\right)^{2} \mathrm{id}
$$

for all $v \in S X$. Hence, the sectional curvature is constant.

\section{The rank of a harmonic manifold}

The notion of rank has been introduced for general spaces of nonpositive curvature by Ballmann, Brin and Eberlein [BBE] and is one of the central concepts in rigidity theory. This notion can be easily generalized to manifolds without conjugate points.

Definition 3.1. Let $M$ be a manifold without conjugate points. The rank of $v \in S M$ is defined by

$$
\operatorname{rank}(v)=\operatorname{dim} \mathscr{L}(v)+1,
$$

where $\mathscr{L}(v)=\operatorname{ker}\left(U_{v}^{\prime}(0)-S_{v}^{\prime}(0)\right)$. The rank of $M$ is defined to be

$$
\operatorname{rank}(M)=\min \{\operatorname{rank}(v) \mid v \in S M\} .
$$

Remark. In the case of nonpositive curvature (or more generally no focal points as shown in Section 6) the rank $(v)$ equals the dimension of parallel Jacobi fields along the geodesic $c_{v}$. However, in general such a relation does not hold. See [Bu] for an explicit example and [Kn2] for general results on manifolds without conjugate points. 
From Corollary 2.5 we immediately obtain:

Corollary 3.2. Let $X$ be a noncompact simply connected harmonic manifold. Then $X$ has purely exponential volume growth if an only if the rank of $X$ is one.

The following lemma is well known.

Lemma 3.3. Let $M$ be a manifold without conjugate points whose sectional curvature is bounded from below by $-\beta^{2}$ for some $\beta \geq 0$. Then

$$
\left|\left\langle U_{v}^{\prime}(0) x, x\right\rangle\right| \leq \beta\langle x, x\rangle \text { and }\left|\left\langle S_{v}^{\prime}(0) x, x\right\rangle\right| \leq \beta\langle x, x\rangle
$$

for all $v \in S M$ and $x \in v^{\perp}$.

Proof. For a proof see for instance [Kn].

We also will need the following result of J. Bolton [Bo] which provides a sufficient condition for a manifold without conjugate points that their geodesic flow is Anosov. In the compact case this result has been obtained by P. Eberlein [Eb]. In the noncompact case one has to specify a metric in order to define the Anosov condition. A natural metric is the Sasaki metric. Using the isomorphism

$$
\left(d \pi_{v}, C_{v}\right): T_{v} T M \rightarrow T_{\pi v} M \times T_{\pi v} M \xi \rightarrow\left(d \pi_{v}(\xi), C_{v}(\xi)\right)=\left(\xi_{1}, \xi_{2}\right),
$$

where $\pi: T M \rightarrow M$ is the canonical projection and $C_{v}: T_{v} T M \rightarrow T_{\pi v} M$ is the connection map, one defines the Sasaki metric via

$$
\langle\xi, \eta\rangle:=\left\langle\xi_{1}, \eta_{1}\right\rangle+\left\langle\xi_{2}, \eta_{2}\right\rangle
$$

Then the geodesic flow $\phi^{t}: S M \rightarrow S M$ is Anosov with respect to the Sasaki metric if there exists a splitting

$$
T_{v} S M=E^{s}(v) \oplus E^{u}(v) \oplus E^{c}(v)
$$

and constants $a \geq 1$ and $b>0$ such that for all $\xi \in E^{s}(v)$,

$$
\left\|D \phi^{t}(v) \xi\right\| \leq a\|\xi\| e^{-b t}, \quad t \geq 0, \quad \text { and } \quad\left\|D \phi^{t}(v) \xi\right\| \geq \frac{1}{a}\|\xi\| e^{-b t}, \quad t \leq 0,
$$

and for all $\xi \in E^{u}(v)$,

$$
\left\|D \phi^{t}(v) \xi\right\| \geq \frac{1}{a}\|\xi\| e^{b t}, \quad t \geq 0, \quad \text { and } \quad\left\|D \phi^{t}(v) \xi\right\| \leq a\|\xi\| e^{b t}, \quad t \leq 0 .
$$

Theorem 3.4. Let $M$ be a manifold without conjugate points and sectional curvature bounded from below. Then the geodesic flow $\phi^{t}: S M \rightarrow S M$ is Anosov if and only if there exists a constant $\rho>0$ such that

$$
\left\langle\left(U_{v}^{\prime}(0)-S_{v}^{\prime}(0)\right) x, x\right\rangle \geq \rho\langle x, x\rangle
$$

for all $x \in v^{\perp}$. 
We recall that W. Klingenberg [Kl] and R. Mañé [Ma] (in a more general setting) proved that Riemannian metrics on compact manifolds do not have conjugate points if their geodesic flow is Anosov.

Theorem 3.5. Let $X$ be a noncompact simply connected harmonic manifold. Then the geodesic flow $\phi^{t}: S X \rightarrow S X$ is Anosov if and only if $\operatorname{rank}(X)=1$.

Proof. Assume that $\operatorname{rank}(X)=1$. Since by Corollary 2.5 the determinant of $\left(U_{v}^{\prime}(0)-\right.$ $\left.S_{v}^{\prime}(0)\right)$ is independent of $v \in S X$, we have $\operatorname{rank}(v)=1$ for all $v \in S X$. By Proposition 6.57 in $[\mathrm{Be}]$ the sectional curvature of a harmonic manifold is bounded. Therefore, Lemma 3.3 implies that the eigenvalues of the nonnegative endomorphism $\left(U_{v}^{\prime}(0)-S_{v}^{\prime}(0)\right)$ are uniformly bounded from above. Since $\operatorname{det}\left(U_{v}^{\prime}(0)-S_{v}^{\prime}(0)\right)=$ const $>0$ the smallest eigenvalue of $\left(U_{v}^{\prime}(0)-S_{v}^{\prime}(0)\right)$ is bounded from below. Hence, we conclude from Theorem 3.4 that the geodesic flow is Anosov. The inverse assertion is a trivial consequence of Theorem 3.4.

The Anosov condition on harmonic manifolds admitting a compact quotient becomes particulary interesting if we combine it with the rigidity of $[\mathrm{BCG}]$ together with [BFL] and [FL].

Theorem 3.6. Let $(M, g)$ be a compact Riemannian manifold such that the geodesic flow is Anosov. Assume that the mean curvature of the horospheres is constant. Then $(M, g)$ is isometric to a locally symmetric space $\left(M_{0}, g_{0}\right)$ of negative curvature.

Proof. From the work of P. Foulon and F. Labourie [FL] follows that the stable and unstable distribution $E^{s}$ and $E^{u}$ of an Anosov geodesic flow are $C^{\infty}$ provided the mean curvature of the horospheres is constant. The results of Y. Benoist, P. Foulon and F. Labourie imply that the geodesic flow on the unit tangent bundle of $(M, g)$ is smoothly conjugate to the geodesic flow on the unit tangent bundle of a locally symmetric space $\left(M_{0}, g_{0}\right)$ of negative curvature. Furthermore, $M, M_{0}$ are homotopy equivalent and the topological entropy as well as the volume of both manifolds $(M, g)$ and $\left(M_{0}, g_{0}\right)$ coincide. Since by a result of A. Freiré and R. Mañé [FM] the volume entropy and the topological entropy for metrics without conjugate points coincide, the work of G. Besson, G. Courtois and S. Gallot implies that $(M, g)$ and $\left(M_{0}, g_{0}\right)$ are isometric.

We immediately obtain:

Corollary 3.7. Let $X$ be a noncompact simply connected harmonic manifold with $\operatorname{rank}(X)=1$. If $X$ admits a compact quotient, then $X$ is a symmetric space of negative curvature. 


\section{Gromov hyperbolic harmonic manifolds}

In this section we will show that for noncompact harmonic manifolds purely exponential volume growth is equivalent to Gromov hyperbolicity.

Definition 4.1. Let $(X, d)$ be a metric space. A curve $c: I \rightarrow X$ defined on an interval $I \subset \mathbb{R}$ is called a geodesic, if $c$ is an isometry, i.e., $d(c(t), c(s))=|t-s|$ for $t, s \in I$. A geodesic metric space $(X, d)$ is a metric space, where each pair of points can be joint by a geodesic.

Remark. Note that in Riemannian geometry geodesics are local isometries. Geodesics in the sense of metric spaces correspond to minimal geodesics in Riemannian geometry.

There are several equivalent definitions of Gromov hyperbolicity. The most common definition is the following.

Definition 4.2. Let $\delta$ be a non negative number. A geodesic metric space is called $\delta$-hyperbolic if all geodesic triangles are $\delta$-thin, i.e., each side of a geodesic triangle is contained in the $\delta$-neighborhood of the two other sides. A geodesic metric space is called Gromov hyperbolic if it is $\delta$-hyperbolic for some $\delta \geq 0$.

We want to show that Gromov hyperbolic harmonic manifolds have purely exponential volume growth. For that we will need the following elementary lemmata.

Lemma 4.3. Let $X$ be a simply connected manifold without conjugate points and $c_{1}, c_{2}: \mathbb{R} \rightarrow X$ be geodesics with $c_{1}(0)=c_{2}(0)$ and $d\left(c_{1}( \pm \ell), c_{2}(\mp \ell)\right) \leq 1$ for $\ell>\frac{1}{2}$. Then

$$
d\left(c_{1}(t), c_{2}(s)\right) \geq 2 \ell-1 \text { for all } s, t \geq \ell .
$$

Proof. Consider $t, s \geq \ell$ and assume $s \leq t$. Then

$$
\begin{aligned}
\ell+t & =d\left(c_{1}(-\ell), c_{1}(t)\right) \\
& \leq d\left(c_{1}(-\ell), c_{2}(\ell)\right)+d\left(c_{2}(\ell), c_{2}(s)\right)+d\left(c_{2}(s), c_{1}(t)\right) \\
& \leq 1+s-\ell+d\left(c_{2}(s), c_{1}(t)\right)
\end{aligned}
$$

and, therefore,

$$
2 \ell-1 \leq d\left(c_{2}(s), c_{1}(t)\right) .
$$

If $s \geq t$, then

$$
\begin{aligned}
\ell+s & =d\left(c_{2}(-\ell), c_{2}(s)\right) \\
& \leq d\left(c_{2}(-\ell), c_{1}(\ell)\right)+d\left(c_{1}(\ell), c_{1}(t)\right)+d\left(c_{1}(t), c_{1}(s)\right) \\
& \leq 1+t-\ell+d\left(c_{1}(t), c_{2}(s)\right)
\end{aligned}
$$

and the assertion follows in this case as well. 
Lemma 4.4. Let $X$ be a simply connected $\delta$-hyperbolic manifold without conjugate points, $p \in X$ and $c:[0, a] \rightarrow X$ a geodesic. Let $c_{1}:\left[0, a_{1}\right] \rightarrow X$ be the geodesic joining $p$ and $c(0)$ and $c_{2}:\left[0, a_{2}\right] \rightarrow X$ be the geodesic joining $p$ and $c(a)$. Then there exist $t_{1} \in\left[0, a_{1}\right], t_{2} \in\left[0, a_{2}\right]$ and $t_{0} \in[0, a]$ such that

$$
d\left(c_{1}\left(t_{1}\right), c\left(t_{0}\right)\right)=d\left(c_{2}\left(t_{2}\right), c\left(t_{0}\right)\right) \leq \delta .
$$

Proof. Consider the continuous function $f:[0, a] \rightarrow \mathbb{R}$ given by

$$
f(t)=d\left(c(t), c_{1}\left[0, a_{1}\right]\right)-d\left(c(t), c_{2}\left[0, a_{2}\right]\right) .
$$

Therefore, $f(0)<0$ and $f(a)>0$ which implies the existence of $t_{0} \in[0, a]$ with

$$
0=f\left(t_{0}\right)=d\left(c\left(t_{0}\right), c_{1}\left[0, a_{1}\right]\right)-d\left(c\left(t_{0}\right), c_{2}\left[0, a_{2}\right]\right) .
$$

Since by assumption geodesic triangles are $\delta$-thin, we have

$$
\left.d\left(c\left(t_{0}\right), c_{1}\left[0, a_{1}\right]\right) \cup c_{2}\left[0, a_{2}\right]\right) \leq \delta,
$$

and we obtain

$$
d\left(c\left(t_{0}\right), c_{1}\left[0, a_{1}\right]\right)=d\left(c\left(t_{0}\right), c_{2}\left[0, a_{2}\right]\right) \leq \delta
$$

which implies the assertion of the lemma.

Corollary 4.5. Let $X$ be a simply connected $\delta$-hyperbolic manifold without conjugate points. Let $c_{1}, c_{2}: \mathbb{R} \rightarrow X$ be geodesics with $c_{1}(0)=c_{2}(0)=p$ and $d\left(c_{1}( \pm \ell), c_{2}(\mp \ell)\right) \leq 1$, where $\ell:=\delta+1$. For $a_{1}, a_{2}>\ell$ consider the geodesic $c:[0, a] \rightarrow X$ joining $c_{1}\left(a_{1}\right)$ and $c_{2}\left(a_{2}\right)$. Then there exists $t_{0} \in[0, a]$ such that

$$
d\left(p, c\left(t_{0}\right)\right) \leq 2 \delta+1 .
$$

Proof. By Lemma 4.4 there exist $t_{1} \in c_{1}\left[0, a_{1}\right], t_{2} \in c_{2}\left[0, a_{2}\right]$ and $t_{0} \in c[0, a]$ such that

$$
d\left(c_{1}\left(t_{1}\right), c\left(t_{0}\right)\right)=d\left(c_{2}\left(t_{2}\right), c\left(t_{0}\right)\right) \leq \delta
$$

and, therefore, $d\left(c_{1}\left(t_{1}\right), c_{2}\left(t_{2}\right)\right) \leq 2 \delta=2 \ell-2$. Then $\min \left(t_{1}, t_{2}\right) \leq \delta+1=\ell$ since otherwise Lemma 4.3 would imply that $d\left(c_{1}\left(t_{1}\right), c_{2}\left(t_{2}\right)\right) \geq 2 \ell-1$ which obviously is a contradiction. Assume $0 \leq t_{1} \leq \delta+1$, we obtain

$$
d\left(p, c\left(t_{0}\right)\right) \leq d\left(p, c_{1}\left(t_{1}\right)\right)+d\left(c_{1}\left(t_{1}\right), c\left(t_{0}\right)\right) \leq 2 \delta+1
$$

which yields the assertion.

Let $X$ be a simply connected manifold without conjugate points and $v \in S_{p} X$. Consider for $t \geq 0$ the function $b_{v, t}(q)=d\left(q, c_{v}(t)\right)-t$. Then for all $q \in X$ the limit

$$
b_{v}(q)=\lim _{t \rightarrow \infty} b_{v, t}(q)
$$


exists and defines the Busemann function $b_{v}$ associated to the geodesic $c_{v}$. The levels of the Busemann functions are the horospheres. It is easy to see that $b_{v}$ is a $C^{1}$ function [Es] with $\left\|\operatorname{grad} b_{v}\right\|=1$ and one can even prove [Kn] that they are of class $C^{1,1}$, i.e., the grad $b_{v}$ is Lipschitz. This implies that the integral curves of $\operatorname{grad} b_{v}$ are geodesics and $\left|b_{v}(q)-b_{v}(p)\right| \leq d(p, q)$. In the case of simply connected noncompact harmonic manifold one can show [RSh3] that Busemann functions are analytic. Note, that $\Delta b_{v}=h$, where $h$ is the mean curvature of the horospheres.

Corollary 4.6. Let $X$ be a simply connected $\delta$-hyperbolic manifold without conjugate points. Consider for $v \in S_{p} X, \ell=\delta+1$ and $r>0$ the spherical cone in $X$ given by

$$
A_{v, \ell}(r):=\left\{c_{w}(t) \mid 0 \leq t \leq r, w \in S_{p} X, d\left(c_{v}( \pm \ell), c_{w}( \pm \ell)\right) \leq 1\right\} .
$$

Then, for $\rho=4 \delta+2$ the set $A_{v, \ell}(r)$ is contained in

$$
\begin{aligned}
H_{v, \rho}(r):=\left\{c_{q}(t) \mid\right. & -\rho / 2 \leq t \leq r, c_{q} \text { is an integral curve of } \\
& \left.\operatorname{grad} b_{-v} \text { with } c_{q}(0)=q \in b_{-v}^{-1}(0) \cap B(p, \rho)\right\}
\end{aligned}
$$

Proof. For $c_{w}(t) \in A_{v, \ell}(r)$ there is a unique integral curve $c_{q}: \mathbb{R} \rightarrow X$ of $\operatorname{grad} b_{-v}$ such that $c_{q}(0)=q \in b_{-v}^{-1}(0)$ and $c_{q}(a)=c_{w}(t)$ for some $a \in \mathbb{R}$. Let $c_{q, s}$ be the sequence of geodesics with $c_{q, s}(a)=c_{q}(a)$ and $c_{q, s}\left(b_{s}\right)=c_{-v}(s)$ for $b_{s} \leq a$. Since

$$
d\left(c_{-v}(\mp \ell), c_{w}( \pm \ell)\right)=d\left(c_{v}( \pm \ell), c_{w}( \pm \ell)\right) \leq 1,
$$

Corollary 4.5 implies the existence of $x_{s} \in c_{q, s}\left(\left[b_{s}, a\right]\right)$ such that $d\left(x_{s}, p\right) \leq 2 \delta+1$. Note, that $c_{q}=\lim _{s \rightarrow \infty} c_{q, s}$. Hence, there also exists $t_{0} \leq a$ such that $d\left(c_{q}\left(t_{0}\right), p\right) \leq$ $2 \delta+1$. Since $b_{-v}\left(c_{q}(t)\right)=t$ we obtain

$$
\left|t_{0}\right|=\left|b_{-v}\left(c_{q}\left(t_{0}\right)\right)\right|=\left|b_{-v}\left(c_{q}\left(t_{0}\right)\right)-b_{-v}(p)\right| \leq d\left(c_{q}\left(t_{0}\right), p\right) \leq 2 \delta+1
$$

and

$$
|a|=\left|b_{-v}\left(c_{q}(a)\right)\right|=\left|b_{-v}\left(c_{w}(t)\right)-b_{-v}(p)\right| \leq d\left(c_{w}(t), p\right) \leq t .
$$

Therefore,

$$
d(p, q)=d\left(p, c_{q}(0)\right) \leq d\left(p, c_{q}\left(t_{0}\right)\right)+d\left(c_{q}\left(t_{0}\right), c_{q}(0)\right) \leq 4 \delta+2,
$$

and $-2 \delta-1 \leq t_{0} \leq a \leq t \leq r$.

Now we can prove the following important proposition.

Proposition 4.7. Let $X$ be a simply connected noncompact harmonic manifold and assume that $X$ is $\delta$-hyperbolic for some $\delta>0$. Then $X$ has purely exponential volume growth. 
Proof. Consider for $\ell=\delta+1$ and $v \in S_{p} X$ the set

$$
A_{v, \ell}(r):=\left\{c_{w}(t) \mid 0 \leq t \leq r, w \in S_{p} X, d\left(c_{v}( \pm \ell), c_{w}( \pm \ell)\right) \leq 1\right\} .
$$

Then

$$
\operatorname{vol}\left(A_{v, \ell}(r)\right)=\int_{0}^{r} f(s) d s \mu_{p}\left(C_{v, \ell}\right)
$$

where

$$
C_{v, \ell}:=\left\{w \in S_{p} X \mid d\left(c_{v}( \pm \ell), c_{w}( \pm \ell)\right) \leq 1\right\}
$$

and $\mu_{p}$ denotes the measure on the sphere $S_{p} X$ induced by Riemannian metric. Corollary 4.6 implies that for $\rho=4 \delta+2$ the set $A_{v, \ell}(r)$ is contained in

$$
\begin{aligned}
H_{v, \rho}(r):=\left\{c_{q}(t) \mid\right. & -\rho / 2 \leq t \leq r, c_{q} \text { is an integral curve of } \\
& \left.\operatorname{grad} b_{-v} \text { with } c_{q}(0)=q \in b_{-v}^{-1}(0) \cap B(p, \rho)\right\} .
\end{aligned}
$$

Furthermore,

$$
\begin{aligned}
\operatorname{vol}\left(H_{v, \rho}(r)\right) & =\int_{-\rho / 2}^{r} e^{h s} d s \operatorname{vol}_{0}\left(b_{v}^{-1}(0) \cap B(p, \rho)\right) \\
& \leq \frac{e^{h r}}{h} \operatorname{vol}_{0}\left(b_{v}^{-1}(0) \cap B(p, \rho)\right),
\end{aligned}
$$

where vol $_{0}$ denotes the induced volume on the horosphere $b_{v}^{-1}(0)$. Therefore,

$$
\int_{0}^{r} f(s) d s \mu_{p}\left(C_{v, \ell}\right) \leq \frac{e^{h r}}{h} \operatorname{vol}_{0}\left(b_{v}^{-1}(0) \cap B(p, \rho)\right)
$$

and the ratio

$$
\frac{\int_{0}^{r} f(s) d s}{e^{h r}}
$$

is bounded above by a constant. Therefore, by L'Hospital the ratio

$$
\frac{f(r)}{e^{h r}}
$$

is bounded from above as well and Corollary 2.5 implies that $X$ has purely exponential volume growth.

Now we are able to prove the following main result on simply connected noncompact harmonic manifolds stated in the introduction. 
Theorem 4.8. Let $X$ be a simply connected and noncompact harmonic manifold. Then the following assertions are equivalent:

(i) $X$ is Gromov hyperbolic.

(ii) X has purely exponential volume growth.

(iii) $X$ has rank one.

(iv) $X$ has an Anosov geodesic flow with respect to the Sasaki metric.

Proof. By the previous proposition (i) implies (ii). The equivalence of (ii), (iii) was proved in Corollary 3.2 and the equivalence of (iii) and (iv) has been obtained in Theorem 3.5.

Assume now that the geodesic flow $\phi^{t}: S X \rightarrow S X$ is Anosov with respect to the Sasaki metric. For $v \in S X$ consider the Jacobi tensor with $A_{v}(0)=0$ and $A_{v}^{\prime}(0)=\mathrm{id}$. Then the Anosov condition implies (see [Bo])

$$
\left\|A_{v}(t) x\right\| \geq\|x\| e^{\alpha t} .
$$

Consider two distinct geodesic rays $c_{1}:[0, \infty) \rightarrow X$ and $c_{2}:[0, \infty) \rightarrow X$ with $c_{1}(0)=c_{2}(0)=q$ and define

$$
\begin{aligned}
d_{t}^{q}\left(c_{1}(t), c_{2}(t)\right):=\inf \{L(\gamma) \mid & \gamma:[a, b] \rightarrow X \backslash B(q, t) \text { a piecewise } \\
& \text { smooth curve joining } \left.c_{1}(t) \text { and } c_{2}(t)\right\} .
\end{aligned}
$$

Then

$$
\liminf _{t \rightarrow \infty} \frac{d_{t}^{q}\left(c_{1}(t), c_{2}(t)\right)}{t} \geq \alpha
$$

This implies, using Proposition 1.26 in Chapter III of [BH] that $X$ is Gromov hyperbolic.

The following theorem was known in the case of negative curvature.

Theorem 4.9. Let $M$ be a compact harmonic manifold with noncompact universal cover and with Gromov hyperbolic fundamental group. Then $M$ is a locally symmetric space of negative sectional curvature.

Proof. We first recall that Gromov hyperbolicity is preserved under quasi-isometries (see for instance $[\mathrm{BH}]$ ). Since for compact manifolds the fundamental group with respect to the word-metric is quasi-isometric to its universal cover, the universal cover is Gromov hyperbolic as well. Hence, by the previous theorem the geodesic flow is Anosov and, therefore, Theorem 3.6 implies that $M$ is a locally symmetric space of negative curvature.

Remark. In particular, compact manifolds which admit a metric of negative curvature cannot carry any harmonic metric besides locally symmetric metrics. 
We strongly believe that noncompact harmonic, nonflat manifolds with $h>0$ and higher rank do not exist. The main purpose of the next section is to prove this under the assumption of no focal points.

\section{Harmonic manifolds with bounded asymptote}

In this section, we show now that for a harmonic manifold of bounded asymptote the rank is constant, i.e., independent of the geodesic. In odd dimensions this implies by a result of Steenrod and Whitehead [SW] that the rank is one.

Definition 5.1. Let $M$ be a manifold without conjugate points and $\alpha \geq 1$. A geodesic $c_{v}: \mathbb{R} \rightarrow M$ is called $\alpha$-stable if

$$
\left\|S_{v}(t) x_{t}\right\| \leq \alpha\|x\| \quad \text { and } \quad\left\|U_{v}(t) x_{t}\right\| \geq \frac{1}{\alpha}\|x\|,
$$

for all $t \geq 0$ and parallel vector fields $x_{t}$ with $x_{0}=x \in v^{\perp}$. We call a geodesic stable if it is $\alpha$-stable for some constant $\alpha \geq 1$.

$M$ is called of bounded asymptote if there is a uniform constant $\alpha \geq 1$ for which all geodesics are $\alpha$-stable.

Remark. The notion of bounded asymptote has been introduced by J.-H. Eschenburg [Es]. In particular, if $M$ has nonpositive curvature or, more generally, no focal points, each geodesic is 1 -stable.

Proposition 5.2. Let $M$ be an n-dimensional manifold without conjugate points. Suppose there exists an $\alpha(v)$-stable geodesic $c_{v}$ with $\operatorname{rank}(v)=k+1 \geq 2$. Then for all $x \in \operatorname{ker}\left(U_{v}^{\prime}(0)-S_{v}^{\prime}(0)\right)$

$$
\frac{1}{\alpha^{2}(v) t}\langle x, x\rangle \leq\left\langle\left(U_{v}^{\prime}(0)-S_{v, t}^{\prime}(0)\right) x, x\right\rangle \leq \frac{\alpha^{2}(v)}{t}\langle x, x\rangle .
$$

Let

$$
\lambda_{1}(v, t) \leq \cdots \leq \lambda_{k}(v, t) \leq \lambda_{k+1}(v, t) \leq \cdots \leq \lambda_{n-1}(v, t)
$$

be the eigenvalues of $U_{v}^{\prime}(0)-S_{v, t}^{\prime}(0)$ and

$$
\beta_{t}(v):=\lambda_{k+1}(v, t) \ldots \lambda_{n-1}(v, t),
$$

then $\beta_{t}(v)$ is monotonically decreasing and converging to the product of the positive eigenvalues $\beta(v)$ of $U_{v}^{\prime}(0)-S_{v}^{\prime}(0)$. Furthermore,

$$
\frac{\beta(v)}{\alpha^{2 k}(v)} \leq \operatorname{det}\left(U_{v}^{\prime}(0)-S_{v, t}^{\prime}(0)\right) t^{k} \leq \alpha^{2 k}(v) \beta_{t}(v) .
$$


Proof. Let $\alpha(v) \geq 1$ and $c_{v}$ is an $\alpha(v)$-stable geodesic. Since Lemma 2.3 implies

$$
\left(U_{v}^{\prime}(0)-S_{v, t}^{\prime}(0)\right)=\left(\int_{0}^{t}\left(U_{v}^{*} U_{v}\right)^{-1}(u) d u\right)^{-1}
$$

we have for each unit vector $x \in v^{\perp}$,

$$
\begin{aligned}
\left\langle\left(U_{v}^{\prime}(0)-S_{v, t}^{\prime}(0)\right) x, x\right\rangle & \geq \frac{1}{\max \left\{\int_{0}^{t}\left\langle\left(U_{v}^{*} U_{v}\right)^{-1}(u) y_{u}, y_{u}\right\rangle d u \mid\|y\|=1\right\}} \\
& =\frac{1}{\left\|\int_{0}^{t}\left(U_{v}^{*} U_{v}\right)^{-1}(u) d u\right\|} .
\end{aligned}
$$

Using

$$
\left\|\int_{0}^{t}\left(U_{v}^{*} U_{v}\right)^{-1}(u) d u\right\| \leq \int_{0}^{t}\left\|\left(U_{v}^{*} U_{v}\right)^{-1}(u)\right\| d u \leq \int_{0}^{t}\left\|U_{v}^{-1}(u)\right\|^{2} d u
$$

and

$$
\left\|U_{v}^{-1}(u)\right\|=\frac{1}{\min \left\{\left\|U_{v}(u) x_{u}\right\| \mid\|x\|=1\right\}} \leq \alpha(v),
$$

we obtain

$$
\frac{1}{\alpha^{2}(v) t} \leq\left\langle\left(U_{v}^{\prime}(0)-S_{v, t}^{\prime}(0)\right) x, x\right\rangle .
$$

On the other hand, Lemma 2.3 implies

$$
\left(S_{v}^{\prime}(0)-S_{v, t}^{\prime}(0)\right)=\left(\int_{0}^{t}\left(S_{v}^{*} S_{v}\right)^{-1}(u) d u\right)^{-1}
$$

as well. Using the estimate (7.2), we obtain for all unit vectors $x \in v^{\perp}$ that

$$
\begin{aligned}
\left\langle\left(S_{v}^{\prime}(0)-S_{v, t}^{\prime}(0)\right) x, x\right\rangle & \leq\left\|\left(\int_{0}^{t}\left(S_{v}^{*} S_{v}\right)^{-1}(u) d u\right)^{-1}\right\| \\
& \leq\left(\int_{0}^{t}\left\|\left(S_{v}^{*} S_{v}\right)(u)\right\|^{-1} d u\right)^{-1}=\left(\int_{0}^{t}\left\|S_{v}(u)\right\|^{-2} d u\right)^{-1} \\
& \leq\left(\int_{0}^{t} \frac{1}{\alpha^{2}(v)} d u\right)^{-1}=\frac{\alpha^{2}(v)}{t} .
\end{aligned}
$$


Therefore,

$$
\left\langle\left(S_{v}^{\prime}(0)-S_{v, t}^{\prime}(0)\right) x, x\right\rangle \leq \frac{\alpha^{2}(v)}{t} .
$$

Putting both inequalities together, for all $x \in \operatorname{ker}\left(U_{v}^{\prime}(0)-S_{v}^{\prime}(0)\right)$ with $\|x\|=1$ we obtain

$$
\frac{1}{\alpha^{2}(v) t} \leq\left\langle\left(U_{v}^{\prime}(0)-S_{v, t}^{\prime}(0)\right) x, x\right\rangle \leq \frac{\alpha^{2}(v)}{t},
$$

which implies the first assertion. Let $0<\lambda_{1}(v, t) \leq \cdots \leq \lambda_{n-1}(v, t)$ the eigenvalues of $\left(U_{v}^{\prime}(0)-S_{v, t}^{\prime}(0)\right)$ and $k=\operatorname{dim}\left(\operatorname{ker}\left(U_{v}^{\prime}(0)-S_{v}^{\prime}(0)\right)\right.$. Then using the above estimates and the minimax characterization of eigenvalues we conclude that

$$
\frac{1}{\alpha^{2}(v) t} \leq \lambda_{i}(v, t) \leq \frac{\alpha^{2}(v)}{t}
$$

for $1 \leq i \leq k$. The remaining eigenvalues $\lambda_{k+1}(v, t) \leq \cdots \leq \lambda_{n-1}(v, t)$ of $\left(U_{v}^{\prime}(0)-S_{v, t}^{\prime}(0)\right)$ are monotonically decreasing in $t$ and converging to the positive eigenvalues of $\left(U_{v}^{\prime}(0)-S_{v}^{\prime}(0)\right)$. Hence,

$$
\frac{\beta(v)}{\alpha^{2 k}(v) t^{k}} \leq \operatorname{det}\left(U_{v}^{\prime}(0)-S_{v, t}^{\prime}(0)\right) \leq \frac{\alpha^{2 k}(v) \beta_{t}(v)}{t^{k}},
$$

where $\beta_{t}(v)=\lambda_{k+1}(v, t) \ldots \lambda_{n-1}(v, t)$ and $\beta(v)=\lambda_{k+1}(v) \ldots \lambda_{n-1}(v)$ is the product of the positive eigenvalues.

Corollary 5.3. Let $X$ be a nonflat, noncompact and simply connected harmonic manifold having a stable geodesic $c_{v}$. Then there is a constant $b \geq 1$ such that

$$
\frac{1}{b} \leq \frac{f(t)}{e^{h t} t^{\operatorname{rank}(v)-1}} \leq b
$$

for all $t \geq 1$.

Proof. Using Corollary 2.5, we have

$$
\frac{e^{h t}}{f(t)}=\operatorname{det}\left(U_{v}^{\prime}(0)-S_{v, t}^{\prime}(0)\right) \text {. }
$$

If $k=\operatorname{rank}(v)-1$ the estimate follows from Proposition 5.2.

Corollary 5.4. Let $X$ be a noncompact simply connected harmonic manifold. Then $\operatorname{rank}(v)$ is constant on the set of initial conditions $v \in S M$ corresponding to stable geodesics. For a fixed $\gamma \geq 1$ consider the set

$$
G_{\gamma}:=\left\{v \in S M \mid c_{v} \text { is an } \alpha(v) \text {-stable geodesic with } \alpha(v) \leq \gamma\right\} .
$$


Then, there exists a constant $\rho>0$ such that

$$
\left\langle\left(U_{v}^{\prime}(0)-S_{v}^{\prime}(0)\right) x, x\right\rangle \geq \rho\langle x, x\rangle
$$

for all $x \in \operatorname{ker}\left(U_{v}^{\prime}(0)-S_{v}^{\prime}(0)\right)^{\perp} \subset v^{\perp}$ and $v \in G_{\gamma}$.

Proof. Since the function $\operatorname{det}\left(U_{v}^{\prime}(0)-S_{v, t}^{\prime}(0)\right)$ is independent of $v \in S X$, Proposition 5.2 implies that $\operatorname{rank}(v)$ is constant for all $v$ corresponding to stable geodesics. Now let $v_{0}$ be a fixed and $v$ an arbitrary vector in $G_{\gamma}$. Then the estimate (5.1) implies

$$
\begin{aligned}
\frac{\beta\left(v_{0}\right)}{\alpha^{2 k}\left(v_{0}\right)} & \leq \operatorname{det}\left(U_{v_{0}}^{\prime}(0)-S_{v_{0}, t}^{\prime}(0)\right) t^{k}=\operatorname{det}\left(U_{v}^{\prime}(0)-S_{v, t}^{\prime}(0)\right) t^{k} \\
& \leq \alpha(v)^{2 k} \beta_{t}(v) \leq \gamma^{2 k} \beta_{t}(v)
\end{aligned}
$$

for all $t \geq 0$ and, therefore,

$$
\frac{\beta\left(v_{0}\right)}{\alpha^{2 k}\left(v_{0}\right)} \leq \gamma^{2 k} \beta(v),
$$

where $\beta(v)=\prod_{i=k+1}^{n-1} \lambda_{i}(v)$ is the product of the positive eigenvalues of $\left(U_{v}^{\prime}(0)-\right.$ $\left.S_{v}^{\prime}(0)\right)$. Since the curvature of $M$ is bounded the eigenvalues of $\left(U_{v}^{\prime}(0)-S_{v}^{\prime}(0)\right)$ are bounded by Lemma 3.3 from above. Since $\beta(v)$ is bounded from below, where the bound only depends on $\gamma, \alpha\left(v_{0}\right)$ and $\beta\left(v_{0}\right)$, the same is true for all the positive eigenvalues of $\left(U_{v}^{\prime}(0)-S_{v}^{\prime}(0)\right)$.

Theorem 5.5. Let $X$ be a nonflat, noncompact and simply connected harmonic manifold of odd dimension. Suppose there exists $p \in X$ such that all geodesics $c_{v}$ with initial conditions $v \in S_{p} X$ are stable. Then $X$ has rank 1.

Proof. Since the $\operatorname{rank}(v)$ is constant for each $v \in S_{p} X$ consider the subspace of $v^{\perp} \cong T_{v} S_{p} X$, given by $\mathscr{L}(v)=\operatorname{ker}\left(U_{v}^{\prime}(0)-S_{v}^{\prime}(0)\right)$. Since the rank is constant it defines a continuous distribution. If the dimension of $X$ is odd, the dimension of the sphere $S_{p} X$ is even. By a result of Steenrod and Whitehead [SW] the distribution must be trivial, i.e., $\operatorname{dim} \mathscr{L}(v)$ is zero or has dimension $n-1$. In the latter case $U_{v}^{\prime}(0)=S_{v}^{\prime}(0)$ and, therefore, $e^{h t}=\operatorname{det} U_{v}(t)=\operatorname{det} S_{v}(t)=e^{-h t}$. But then $h=0$ and $X$ is flat. Hence, $\operatorname{dim} \mathscr{L}(v)$ is zero and $\operatorname{rank}(X)=1$.

\section{Harmonic manifolds without focal points}

In this section, we will show that a nonflat simply connected harmonic manifold $X$ without focal points has rank 1 . For odd dimensions the prove has been given in the previous section. Note that $X$ has no focal points if the second fundamental form of 
horospheres is positive semi-definite or if for all $v \in S X$ the Busemann functions $b_{v}$ are convex. In terms of the stable and unstable Jacobi tensors $S_{v}$ and $U_{v}$ this is equivalent to $U_{v}^{\prime}(0) \geq 0$ and therefore $S_{v}^{\prime}(0)=-U_{-v}^{\prime}(0) \leq 0$ for all $v \in S X$. Most of the properties of manifolds of nonpositive curvature are shared by manifolds without focal points. For instance geodesic spheres are convex since horospheres are convex. Furthermore, the flat strip theorem is true, which asserts that two geodesics $c_{1}$ and $c_{2}$ bound a flat strip if $d\left(c_{1}(t), c_{2}(t)\right) \leq b$ for some $b \geq 0$ and all $t \in \mathbb{R}$. This means that there exists an isometric, totally geodesic imbedding $F:[0, a] \times \mathbb{R} \rightarrow X$ such that $c_{1}(t)=F(0, t)$ and $c_{2}(t)=F(a, t)$ (see [Es] for a proof). In particular, $c_{1}$ and $c_{2}$ are parallel, i.e., $d\left(c_{1}(t), c_{2}(t)\right)=a$.

Lemma 6.1. Let $X$ be a manifold without focal points. Then the following holds:

$$
\begin{aligned}
\mathscr{L}(v) & =\operatorname{ker}\left(U_{v}^{\prime}(0)\right) \cap \operatorname{ker}\left(S_{v}^{\prime}(0)\right) \subset v^{\perp} \\
& =\left\{x \in v^{\perp} \mid x_{t} \text { is a parallel Jacobi field along } c_{v}\right\} .
\end{aligned}
$$

Furthermore $x_{t}, S_{v}(t) x_{t}, U_{v}(t) x_{t} \in \mathscr{L}^{\perp}\left(\phi^{t} v\right)$ for all $x \in \mathscr{L}^{\perp}(v)=\left\{x \in v^{\perp} \mid x \perp\right.$ $\mathscr{L}(v)\}$.

Proof. Since $X$ has no focal points, we have

$$
\left\langle\left(S_{v}^{\prime}(0) x, x\right\rangle \leq 0 \leq\left\langle\left(U_{v}^{\prime}(0) x, x\right\rangle\right.\right.
$$

for all $x \in v^{\perp}$. Due to the fact that $S_{v}^{\prime}(0)$ and $U_{v}^{\prime}(0)$ are symmetric endomorphisms, $x \in \operatorname{ker}\left(U_{v}^{\prime}(0)-S_{v}^{\prime}(0)\right)$ implies $x \in \operatorname{ker}\left(U_{v}^{\prime}(0) \cap \operatorname{ker}\left(S_{v}^{\prime}(0)\right)\right.$.

Assume that $x \in \mathscr{L}(v)$. Then Lemma 7.3 implies $U_{v}(t) x_{t} \in \mathscr{L}\left(\phi^{t} v\right)$ and we obtain

$$
\left(U_{v}(t) x_{t}\right)^{\prime}=U_{v}^{\prime}(t) x_{t}=U_{v}^{\prime}(t) U_{v}^{-1}(t) U_{v}(t) x_{t}=U_{\phi^{t} v}^{\prime}(0) U_{v}(t) x_{t}=0 .
$$

Hence $U_{v}(t) x_{t}$ is parallel along $c_{v}$. In particular, $U_{v}(0)=\mathrm{id}$ yields $U_{v}(t) x_{t}=x_{t}$.

Now consider $x \in v^{\perp}$ such that its parallel translation defines a Jacobi field. Then for each $s \neq 0 J_{s}(t)=\frac{s-t}{s} x_{t}$ defines a Jacobi field with $J_{s}(0)=x$ and $J_{s}(s)=0$. Therefore,

$$
U_{v} x_{t}=\lim _{s \rightarrow \infty} J_{s}(t)=x_{t}=\lim _{s \rightarrow-\infty} J_{s}(t)=S_{v} x_{t} .
$$

Assume that $x \in \mathscr{L}^{\perp}(v)$. Then for all $y \in \mathscr{L}(v)$, we have $\left\langle y_{t}, x_{t}\right\rangle=\langle x, y\rangle$ and $y_{t} \in \mathscr{L}\left(\phi^{t} v\right)$ implies $x_{t} \in \mathscr{L}^{\perp}\left(\phi^{t}(v)\right)$.

To prove the last assertion consider $x \in \mathscr{L}^{\perp}(v)$. Then for all $y \in \mathscr{L}(v)$, we obtain

$$
\begin{aligned}
\left\langle U_{v}(t) x_{t}, y_{t}\right\rangle^{\prime} & =\left\langle U_{v}^{\prime}(t) x_{t}, y_{t}\right\rangle=\left\langle U_{v}^{\prime}(t) U_{v}^{-1}(t) U_{v}(t) x_{t}, y_{t}\right\rangle \\
& =\left\langle U_{\phi^{t} v}^{\prime}(0) U_{v}(t) x_{t}, y_{t}\right\rangle=\left\langle U_{v}(t) x_{t}, U_{\phi^{t} v}^{\prime}(0) y_{t}\right\rangle=0 .
\end{aligned}
$$

Hence, $\left\langle U_{v}(t) x_{t}, y_{t}\right\rangle=\langle x, y\rangle=0$ and, therefore, $U_{v}(t) x_{t} \in \mathscr{L}^{\perp}\left(\phi^{t}(v)\right)$. In the same way one proves: $S_{v}(t) x_{t} \in \mathscr{L}^{\perp}\left(\phi^{t}(v)\right)$. 
Lemma 6.2. Let $X$ be a simply connected manifold without focal points such that $\operatorname{dim} \mathscr{L}(v)$ is positive and independent of $v$. Then, for each compact set $K \subset S X$ there exists $T>0$ such that

$$
\mathscr{L}(v)=\left\{x \in v^{\perp} \mid R\left(x_{t}, \dot{c}_{v}(t), \dot{c}_{v}(t)\right)=0, t \in[-T, T]\right\} .
$$

for all $v \in K$. In particular, $\mathscr{L}(v)$ and $\mathscr{L}^{\perp}(v)$ depend smoothly on $v \in S X$.

Proof. According to Lemma 6.1 we have that $x \in \mathscr{L}(v)$ if and only if its parallel translation $x_{t}$ is a Jacobi field along $c_{v}$. Therefore, each $x \in \mathscr{L}(v)$ is contained in

$$
\mathscr{L}_{T}(v):=\left\{x \in v^{\perp} \mid R\left(x_{t}, \dot{c}_{v}(t)\right) \dot{c}_{v}(t)=0, t \in[-T, T]\right\}
$$

for all $T>0$. Let $K \subset S X$ be compact. If $T$ with the required property would not exist, we could choose a convergent sequence $v_{n} \in K$ and a sequence $T_{n}$ with $T_{n} \rightarrow \infty$ such that for $v=\lim _{n \rightarrow \infty} v_{n}=v$

$$
\operatorname{dim} \mathscr{L}_{T_{n}}\left(v_{n}\right)>\operatorname{dim} \mathscr{L}\left(v_{n}\right)=\operatorname{dim} \mathscr{L}(v)
$$

which would imply

$$
\operatorname{dim}\left\{x \in v^{\perp} \mid R\left(x_{t}, \dot{c}_{v}(t), \dot{c}_{v}(t)\right)=0, t \in \mathbb{R}\right\}>\operatorname{dim} \mathscr{L}(v) .
$$

On the other hand, each $x \in v^{\perp}$ with $R\left(x_{t}, \dot{c}_{v}(t), \dot{c}_{v}(t)\right)=0$ for all $t \in \mathbb{R}$ defines a parallel Jacobi field along $c_{v}$ and, hence, is contained in $\mathscr{L}(v)$ which leads to a contradiction. Hence, locally $\mathscr{L}(v)=\mathscr{L}_{T}(v)$ for sufficiently large $T$. In particular, $\mathscr{L}_{T}(v)$ and $\mathscr{L}^{\perp}(v)$ depend smoothly on $v \in S X$.

Proposition 6.3. Let $X$ be a nonflat manifold without focal points and bounded sectional curvature. Assume that the eigenvalues of $\left(U_{v}^{\prime}(0)-S_{v}^{\prime}(0)\right)$ restricted to $\mathscr{L}^{\perp}(v) \subset v^{\perp}$ are bounded from below by a positive constant independent of $v$. Then there are constants $a \geq 1$ and $\alpha>0$ independent of $v$ such that

as well as

$$
\left\|S_{v}(t) x_{t}\right\| \leq a e^{-\alpha t}\|x\| \quad \text { and } \quad\left\|S_{v}(-t) x_{-t}\right\| \geq \frac{1}{a} e^{\alpha t}\|x\|
$$

$$
\left\|U_{v}(t) x_{t}\right\| \geq \frac{1}{a} e^{\alpha t}\|x\| \quad \text { and } \quad\left\|U_{v}(-t) x_{-t}\right\| \geq a e^{-\alpha t}\|x\|
$$

for all $x \in \mathscr{L}^{\perp}(v)$ and $t \geq 0$. Furthermore, for $t \geq 0$,

$$
\left\|A_{v}(t) x_{t}\right\|=t\|x\| \text { for all } x \in \mathscr{L}(v)
$$

and there exists a constant $a^{\prime}$ such that

$$
\left\|A_{v}(t) x_{t}\right\| \geq a^{\prime} e^{\alpha t}\|x\| \quad \text { for all } x \in \mathscr{L}^{\perp}(v)
$$

for all $t \geq 1$. 
Proof. Consider the equation

$$
S_{v}^{*}(t)\left(U_{\phi^{t}(v)}^{\prime}(0)-S_{\phi^{t}(v)}^{\prime}(0)\right) S_{v}^{*}(t)=\left(\int_{-\infty}^{t}\left(S_{v}^{*} S_{v}\right)^{-1}(u) d u\right)^{-1}
$$

proved in Lemma 7.3 of the appendix. Using Lemma 6.1 the left and, therefore, also the right hand side define a strictly positive symmetric endomorphism on $\mathscr{L}^{\perp}\left(\phi^{t} v\right)$. Since the eigenvalues of $\left(U_{v}^{\prime}(0)-S_{v}^{\prime}(0)\right)$ restricted to $\mathscr{L}^{\perp}(v) \subset v^{\perp}$ are bounded from below by a positive constant $\rho$, Lemma 7.3 of the appendix implies

$$
\begin{aligned}
\rho & \leq\left\langle\left(\int_{-\infty}^{t}\left(S_{v}^{*} S_{v}\right)^{-1}(u) d u\right)_{\mathscr{L}^{\perp}\left(\phi^{t} v\right)}^{-1} S_{v}^{-1}(t) x, S_{v}^{-1}(t) x\right\rangle \\
& \leq\left\|\left(\int_{-\infty}^{t}\left(S_{v}^{*} S_{v}\right)^{-1}(u) d u\right)_{\mid L^{\perp}\left(\phi^{t} v\right)}^{-1}\right\|\left\|S_{v}^{-1}(t) x\right\|^{2}
\end{aligned}
$$

for all $x \in \mathscr{L}^{\perp}\left(\phi^{t} v\right)$ with $\|x\|=1$. Furthermore, we have

$$
\begin{gathered}
\left\|\left(\int_{-\infty}^{t}\left(S_{v}^{*} S_{v}\right)^{-1}(u) d u\right)_{\mid \mathscr{L}^{\perp}\left(\phi^{t} v\right)}^{-1}\right\| \\
\quad=\frac{1}{\min \left\{\int_{-\infty}^{t}\left\langle\left(S_{v}^{*} S_{v}\right)^{-1}(u) y_{u}, y_{u}\right\rangle d u \mid y \in \mathscr{L}^{\perp}(v),\|y\|=1\right\}} .
\end{gathered}
$$

Therefore,

$$
\begin{aligned}
& \left.\rho \min \left\{\int_{-\infty}^{t}\left\langle S_{v}^{*-1}(u) y_{u}, S_{v}^{*-1}(u) y_{u}\right\rangle d u \mid y \in \mathscr{L}^{\perp}(v),\|y\|=1\right)\right\} \\
& \quad \leq\left\|S_{v}^{-1}(t) x\right\|^{2} .
\end{aligned}
$$

Defining

$$
\begin{aligned}
\varphi(u) & :=\min \left\{\left\|S_{v}^{*-1}(u) y\right\|^{2} \mid y \in \mathscr{L}^{\perp}\left(\phi^{u} v\right),\|y\|=1\right\} \\
& =\min \left\{\left\|S_{v}^{-1}(u) y\right\|^{2} \mid y \in \mathscr{L}^{\perp}\left(\phi^{u} v\right),\|y\|=1\right\},
\end{aligned}
$$

we obtain

$$
\rho \int_{0}^{t} \varphi(u) d u \leq \rho \int_{-\infty}^{t} \varphi(u) d u \leq \varphi(t)
$$


and, hence,

$$
\rho F(t) \leq F^{\prime}(t)
$$

for $F(t):=\int_{0}^{t} \varphi(u) d u$. This implies $F(t) \geq F(1) e^{\rho t}$ for all $t \geq 1$ and, therefore, $\varphi(t)=F^{\prime}(t) \geq \rho F(t) \geq \rho F(1) e^{\rho t}$ for all $t \geq 1$. Since the sectional curvature of $X$ is bounded, $\varphi(t)$ is on $[0,1]$ bounded away from 0 . Hence, there exist a constant $a \geq 1$ such that

$$
\left\|S_{v}^{-1}(t) y\right\| \geq \frac{1}{a} e^{\alpha t}\|y\|
$$

for $\alpha=\frac{\rho}{2}$ and all $y \in \mathscr{L}^{\perp}\left(\phi^{t} v\right)$ and $t \geq 0$. Since $S_{v}(t): \mathscr{L}^{\perp}\left(\phi^{t} v\right) \rightarrow \mathscr{L}^{\perp}\left(\phi^{t} v\right)$ is an isomorphism, we obtain for all $x \in \mathscr{L}^{\perp}\left(\phi^{t} v\right)$ and $t \geq 0$

$$
\left\|S_{v}(t) x\right\| \leq a e^{-\alpha t}\|x\| .
$$

Note, that for each $u \in \mathbb{R}$, we have

$$
S_{\phi^{u} v}(t) x_{t}=S_{v}(t+u)\left(S_{v}^{-1}(u) x\right)_{t},
$$

where $x_{t}$ is the parallel translation of $x \in \mathscr{L}^{\perp}\left(\phi^{u}(v)\right)$ along $c_{\phi^{u}} v(t)$. Hence, for $t=-u \leq 0$ we obtain with (6.1)

$$
\left\|S_{\phi^{u} v}(-u) x_{-u}\right\|=\left\|\left(S_{v}^{-1}(u) x\right)_{-u}\right\|=\left\|\left(S_{v}^{-1}(u) x\right)\right\| \geq \frac{1}{a} e^{\alpha u}\|x\| .
$$

In particular, for $w=\phi^{u} v$ and $u \geq 0$ the estimate

$$
\left\|S_{w}(-u) x_{-u}\right\| \geq \frac{1}{a} e^{\alpha u}\|x\|
$$

holds for all $x \in \mathscr{L}^{\perp}(w)$. Since $U_{v}(t)=S_{-v}(-t)$ the second estimate of the proposition follows.

To prove the remaining assertions we recall that

$$
A_{v}(t) x_{t}=U_{v}(t) \int_{0}^{t}\left(U_{v}^{*} U_{v}\right)^{-1}(s) x_{s} d s .
$$

If $x \in \mathscr{L}(v)$ we have $\left(U_{v}^{*} U_{v}\right)^{-1}(s) x_{s}=x_{s}$ and, therefore, $A_{v}(t) x_{t}=U_{v}(t)\left(t x_{t}\right)=$ $t x_{t}$.

If $x \in \mathscr{L}^{\perp}(v)$ we have $\int_{0}^{t}\left(U_{v}^{*} U_{v}\right)^{-1}(s) x_{s} d s \in \mathscr{L}^{\perp}\left(\phi^{t} v\right)$. Therefore,

$$
\left\|A_{v}(t) x_{t}\right\| \geq a e^{\alpha t}\left\|\int_{0}^{t}\left(U_{v}^{*} U_{v}\right)^{-1}(s) x_{s} d s\right\| .
$$


Since Lemma 2.3 implies

$$
\left(\left(U_{v}^{\prime}(0)-S_{v, t}^{\prime}(0)\right)^{-1} x\right)_{t}=\int_{0}^{t}\left(U_{v}^{*} U_{v}\right)^{-1}(s) x_{s} d s
$$

and since for $t \geq 1$ there exists a constant $b>0$ such that $\left\langle\left(U_{v}^{\prime}(0)-S_{v, t}^{\prime}(0)\right) x, x\right\rangle \leq$ $b\langle x, x\rangle$ for all $x \in v^{\perp}$ the last estimate follows.

Theorem 6.4. Let X be a nonflat manifold without focal points and bounded sectional curvature. Assume that the rank of $X$ is constant, i.e., the dimension of

$$
\operatorname{dim} \mathscr{L}(v)=\operatorname{dim}\left\{x \in v^{\perp} \mid v \in \operatorname{ker}\left(U_{v}^{\prime}(0)\right) \cap \operatorname{ker}\left(S_{v}^{\prime}(0)\right)=\operatorname{rank}(v)-1\right.
$$

is independent of $v$. Furthermore assume, that there exists a constant $\rho>0$ such that

$$
\left\langle\left(U_{v}^{\prime}(0)-S_{v}^{\prime}(0)\right) x, x\right\rangle \geq \rho\langle x, x\rangle
$$

for all $x \in \mathscr{L}^{\perp}(v)$. Then the rank is equal to one and the geodesic flow is Anosov.

As a consequence we obtain:

Theorem 6.5. Let $X$ be a simply connected nonflat harmonic manifold without focal points. Then $X$ is of rank one. Moreover, the geodesic flow is Anosov and $X$ is Gromov hyperbolic.

Proof. Since $X$ is harmonic and has no focal points Corollary 5.4 implies that the conditions of Theorem 6.4 are fulfilled.

It remains to prove 6.4 .

We first show that the geodesic flow is partially hyperbolic. Consider the distributions

$$
\begin{aligned}
& E^{p}(v)=\{(x+\lambda v, 0) \mid x \in \mathscr{L}(v), \lambda \in \mathbb{R}\}, \\
& E^{c}(v)=\{(x+\lambda v, y) \mid x, y \in \mathscr{L}(v), \lambda \in \mathbb{R}\}, \\
& \left.E^{s}(v)=\left\{x, S_{v}^{\prime}(0) x\right) \mid x \in \mathscr{L}^{\perp}(v)\right\}, \\
& \left.E^{u}(v)=\left\{x, U_{v}^{\prime}(0) x\right) \mid x \in \mathscr{L}^{\perp}(v)\right\} .
\end{aligned}
$$

We call $E^{p}$ the parallel, $E^{c}$ the central, $E^{s}$ the stable and $E^{u}$ the unstable distribution. Obviously $E^{p}(v) \subset E^{c}(v)$. Furthermore, the central, stable and the unstable distributions are transversal and

$$
\operatorname{dim} E^{c}(v)=2 \operatorname{rank}(X)-1, \quad \operatorname{dim} E^{s}(v)=\operatorname{dim} E^{u}(v)=n-\operatorname{rank} X .
$$

Therefore, the sum of the dimension is equal to $2 n-1$ and, hence,

$$
T_{v} S X=E^{c}(v) \oplus E^{s}(v) \oplus E^{u}(v) .
$$


Lemma 6.6. Under the assumption of Theorem 6.4, the geodesic flow is partially hyperbolic with respect to the Sasaki metric. More precisely, there are constants $\alpha>0$, and $b, c \geq 1$ such that for all $\xi \in E^{s}(v)$, we have

$$
\left\|D \phi^{t}(v) \xi\right\| \leq b\|\xi\| e^{-\alpha t}, \quad t \geq 0, \quad \text { and }\left\|D \phi^{t}(v) \xi\right\| \geq \frac{1}{b}\|\xi\| e^{-\alpha t}, \quad t \leq 0 .
$$

Furthermore for all $\xi \in E^{u}(v)$ we have

$$
\left\|D \phi^{t}(v) \xi\right\| \geq \frac{1}{b}\|\xi\| e^{\alpha t}, \quad t \geq 0, \quad \text { and }\left\|D \phi^{t}(v) \xi\right\| \leq b\|\xi\| e^{\alpha t}, \quad t \leq 0 .
$$

For all $\xi \in E^{c}(v)$ and $t \in \mathbb{R}$ we obtain

$$
\left\|D \phi^{t}(v) \xi\right\| \leq c\|\xi\|(|t|+1) .
$$

Proof. For $\xi=\left(x, S_{v}^{\prime}(0) x\right) \in E^{s}(v)$ we obtain

$$
\begin{aligned}
\left\|D \phi^{t}(v) \xi\right\| & =\left\|\left(S_{v}(t) x_{t}, S_{v}^{\prime}(t) x_{t}\right)\right\|=\sqrt{\left.\left\|S_{v}(t) x_{t}\right\|^{2}+\| S_{v}^{\prime}(t) x_{t}\right) \|^{2}} \\
& =\left\|S_{v}(t) x_{t}\right\| \sqrt{1+\frac{\left.\| S_{v}^{\prime}(t) x_{t}\right) \|^{2}}{\left\|S_{v}(t) x_{t}\right\|^{2}}} .
\end{aligned}
$$

Moreover, Lemma 3.3 implies

$$
\frac{\left.\| S_{v}^{\prime}(t) x_{t}\right) \|}{\left\|S_{v}(t) x_{t}\right\|} \leq\left\|S_{v}^{\prime}(t) S_{v}(t)^{-1}\right\|=\left\|S_{\phi^{t} v}^{\prime}(0)\right\| \leq \beta
$$

and Proposition 6.3 yields

$$
\left\|D \phi^{t}(v) \xi\right\| \leq a e^{-\alpha t}\|x\| \sqrt{1+\beta^{2}} \leq a e^{-\alpha t}\|\xi\| \sqrt{1+\beta^{2}} .
$$

The remaining assertions are obtained in a similar way.

As we will see, all the distributions are integrable. Define

$$
P(v)=\left\{w \in S X \mid d\left(c_{w}(t), c_{v}(t)\right) \text { is constant }\right\}
$$

to be the subset of $S X$ consisting of vectors tangent to the parallel geodesics of $c_{v}$ and denote by $F(v):=\pi(P(v))$ its projection on $X$.

Proposition 6.7. Assume that $X$ fulfills the assumption of Theorem 6.4. Then the distributions $E^{p}$ and $E^{c}$ are integrable and provide flow invariant and smooth foliations. The integral manifolds of $E^{p}$ are given by $P(v)$. The projection $F(v):=\pi(P(v))$ of each leave is a $k$-flat. The integral manifolds of $E^{c}$ are given by the unit tangent 
bundles $S F(v)$ of the $k$-flats. The distributions $E^{s}$ and $E^{u}$ are integrable as well and the leaves are the stable and unstable manifolds given by

$$
W^{s}(v)=\left\{w \in S X \mid d\left(c_{v}(t), c_{w}(t)\right) \leq a e^{-c t} d(\pi(v), \pi(w)), t \geq 0\right\}
$$

and

$$
W^{u}(v)=\left\{w \in S X \mid d\left(c_{v}(t), c_{w}(t)\right) \leq a e^{c t} d(\pi(v), \pi(w)), t \leq 0\right\}
$$

for constants $c, a>0$.

Proof. The integrability of $E^{p}$ follows as in Lemma 2.2 in [BBE], which was given under the assumption of nonpositive curvature and compact quotient, but not necessarily constant rank. By Lemma 6.2 the distribution $E^{p}(v)=\{(x+\lambda v, 0) \mid x \in \mathscr{L}(v)\}$ is smooth. Choose a smooth curve $\rho:[0, a] \rightarrow S X$ with $\rho(0)=v$ tangent to $E^{p}$, i.e. $\frac{d}{d s} \rho(s)=(x(s)+\lambda(s) \rho(s), 0) \in E^{p}(\rho(s))$, and, therefore, $x(s) \in \mathscr{L}(\gamma(s))$. Consider for each $t \in \mathbb{R}$ the curve $\gamma_{t}: I \rightarrow S X$ with $\gamma_{t}(s)=c_{\rho(s)}(t)$. Hence,

$$
\frac{d}{d s} \gamma_{t}(s)=J_{\rho(s)}(t),
$$

where $J_{\rho(s)}(t)$ is the parallel Jacobi field with $J_{\rho(s)}(0)=x(s)+\lambda(s) \rho(s)$. Consequently, the length of $\gamma_{t}(I)$ is constant, the distance $d\left(c_{v}(t), c_{\rho(s)}(t)\right.$ is bounded and the geodesic $c_{\rho(s)}(t)$ is parallel to $c_{v}$. If $\xi$ and $\eta$ are two smooth vector fields tangent to $E^{p}$ the commutator $[\xi, \eta]$ is tangent to $E^{p}$ as well. To prove this consider the flows $\varphi_{\xi}$ and $\varphi_{\eta}$. Then for $s$

$$
f\left(s^{2}\right)=\varphi_{\eta}^{-s} \circ \varphi_{\xi}^{-s} \circ \varphi_{\eta}^{s} \circ \varphi_{\xi}^{s}(v)
$$

is parallel to $v$ and, hence,

$$
\left.\frac{d}{d s}\right|_{s=0} f\left(s^{2}\right)=[\xi, \eta](v) \in E^{p}(v) .
$$

Therefore, each leaf of $E^{p}$ though $v$ is a $k$-dimensional submanifold of $S X$, given by $P(v)$. Now the flat strip theorem (see [Es]) implies that the projections $F(v):=$ $\pi(P(v))$ are $k$-flats, i.e., totally geodesic flat spaces isometric to the Euclidean space $\mathbb{R}^{k}$. Therefore, the unit tangent bundle of a $k$-flat is flow invariant and as one easily checks tangent to the central distribution $E^{c}$.

Since $\phi^{t}$ defines a partially hyperbolic flow the stable and unstable distributions $E^{s}$ and $E^{u}$ are integrable and tangent to $W^{s}$ and $W^{u}$.

Let $X$ be a simply connected manifold without conjugate points. Let $B(q, t)$ be the open ball of radius $t$ about $q$. For $q_{1}, q_{2} \in X \backslash B(q, t)$ we call

$$
\begin{aligned}
d_{t}^{q}\left(q_{1}, q_{2}\right):=\inf \{L(\gamma) \mid & \gamma:[a, b] \rightarrow X \backslash B(q, t) \text { piecewise } \\
& \text { smooth curve joining } \left.q_{1} \text { and } q_{2}\right\} .
\end{aligned}
$$


The following lemma is important for the proof of Theorem 6.4. We remark that it does not hold for symmetric of higher rank.

Lemma 6.8. Let $X$ be a simply connected manifold without focal points which fulfills the assumption of Theorem 6.4 and assume that its rank is at least 2. Given $v \in S_{q} X$, then for each $w \in S_{q} X$ the following assertions are equivalent:

(a) $w \in S_{q} F(v)$,

(b) $\varlimsup_{t \rightarrow \infty} \frac{1}{t} d_{t}^{q}\left(c_{v}(t), c_{w}(t)\right)<\infty$,

(c) there exists $\rho \geq 0$ such that $\varlimsup_{t \rightarrow \infty} \frac{1}{t} d_{t-\rho}^{q}\left(c_{v}(t), c_{w}(t)\right)<\infty$.

Proof. We can assume that $X$ is not flat. Assume $w \in S_{q} F(v)$. Consider a shortest curve $x:[0, a] \rightarrow S_{q} F(v)$ such that $x(0)=v, x(a)=w$ and $\left\|x^{\prime}(s)\right\|=1$. In particular, $x^{\prime}(s) \in \mathscr{L}(x(s))$. Then $\gamma_{t}(s)=\exp _{q}(t x(s))$ connects $c_{v}(t)$ and $c_{w}(t)$ and the image is in the complement of $B(q, t)$. Furthermore, $\dot{\gamma}_{t}(s)=J(t)$ is the Jacobi field along the geodesic $c_{x(s)}$ with $J(0)=0$ and $J^{\prime}(0)=x^{\prime}(s)$. Hence, $J(t)=A_{x(s)}(t)\left(x^{\prime}(s)\right)_{t}$, where $A_{x(s)}$ is the Jacobi tensor along $c_{x(s)}$ with $A_{x(s)}(0)$ $=0$ and $A_{x(s)}^{\prime}(0)=$ id. Since $x^{\prime}(s) \in \mathscr{L}(x(s))$ and $\left\|x^{\prime}(s)\right\|=1$ Proposition 6.3 implies

$$
\left\|\dot{\gamma}_{t}(s)\right\|=\left\|A_{x(s)}(t)\left(x^{\prime}(s)\right)_{t}\right\|=t .
$$

Hence, $L\left(\gamma_{t}\right)=t a$ and, therefore, (a) implies (b).

Since for all $\rho \geq 0$ and $q_{1}, q_{2} \in X \backslash B(q, t)$ we have $d_{t-\rho}^{q}\left(q_{1}, q_{2}\right) \leq d_{t}^{q}\left(q_{1}, q_{2}\right)$, assertion (c) follows from (b).

Now assume that (c) holds and $w \notin S_{q} F(v)$. Let $\gamma:[0,1] \rightarrow X$ be a smooth curve such that $\gamma(0)=c_{v}(t), \gamma(1)=c_{w}(t)$ and $d(\gamma(s), q)=f(s) \geq t-\rho$. Consider the curve $x:[0,1] \rightarrow S_{q} X$ such that $\gamma(s)=\exp _{q}(f(s) x(s))$ and the geodesic variation

$$
\varphi(s, u)=\exp _{q}(u x(s)), \quad \text { where } 0 \leq u \leq f(s) .
$$

Then

$$
\frac{\partial}{\partial s} \varphi(s, u)=J_{x(s)}(u)
$$

is the perpendicular Jacobi field along $c_{x(s)}(u)$ with initial conditions $J_{x(s)}(0)=0$ and $J_{x(s)}^{\prime}(0)=x^{\prime}(s)$. Since

$$
\begin{aligned}
\left.\frac{\partial}{\partial s}\right|_{s=s_{0}} \gamma(s) & =\left.\frac{\partial}{\partial s}\right|_{s=s_{0}} \varphi\left(s, f\left(s_{0}\right)\right)+\left.\frac{\partial}{\partial u}\right|_{u=f\left(s_{0}\right)} \varphi\left(s_{0}, u\right) f^{\prime}\left(s_{0}\right) \\
& =J_{x\left(s_{0}\right)}\left(f\left(s_{0}\right)\right)+\dot{c}_{x\left(s_{0}\right)}\left(f\left(s_{0}\right)\right) f^{\prime}\left(s_{0}\right)
\end{aligned}
$$

the estimate

$$
\left\|\left.\frac{\partial}{\partial s}\right|_{s=s_{0}} \gamma(s)\right\| \geq\left\|J_{x\left(s_{0}\right)}\left(f\left(s_{0}\right)\right)\right\|
$$


holds. As above we have $J_{x(s)}(u)=A_{x(s)}(u)\left(x^{\prime}(s)\right)_{u}$. Decompose $x^{\prime}(s)=y(s)+$ $z(s)$, where $y(s) \in \mathscr{L}\left(x(s)\right.$ and $z(s) \in \mathscr{L}^{\perp}\left(x(s)\right.$. Since $w \notin S_{q} F(v)$ there is a constant $b>0$ such $\int_{0}^{1}\|z(s)\| d s \geq b$. Using Proposition 6.3, we obtain

$$
L(\gamma)=\int_{0}^{1}\|\dot{\gamma}(s)\| d s \geq a^{\prime} e^{\alpha(t-\rho)} \int_{0}^{1}\|z(s)\| d s \geq a^{\prime} e^{\alpha(t-\rho)} b
$$

for all $t>0$ in contradiction to (c).

Proof of Theorem 6.4. Assume that the rank of $\mathrm{X}$ is at least 2. Consider $v \in S_{p} X$ and $v^{\prime} \in W^{s}(v)$ where $v^{\prime} \neq v$ and $\pi\left(v^{\prime}\right)=q$. This implies that $d\left(c_{v}(t), c_{v^{\prime}}(t)\right)$ converges to 0 as $t$ tends to $\infty$. For each $w \in S_{p} F(v)$ define $w^{\prime}=-\operatorname{grad} b_{w}(q)$. Since $X$ has no focal points $d\left(c_{w^{\prime}}(t), c_{w}(t)\right) \leq d(p, q)$. In particular, for $\rho=$ $d(p, q)$ we obtain

$$
\begin{gathered}
\frac{1}{t} d_{t}^{q}\left(c_{w^{\prime}}(t), c_{v^{\prime}}(t)\right) \leq \frac{1}{t}\left(d_{t-\rho}^{p}\left(c_{w^{\prime}}(t), c_{w}(t)\right)+d_{t-\rho}^{p}\left(c_{w}(t), c_{v}(t)\right)\right. \\
\left.+d_{t-\rho}^{p}\left(c_{v}(t), c_{v^{\prime}}(t)\right)\right)
\end{gathered}
$$

is bounded for $t \geq 0$. Using Lemma 6.8 this implies $w^{\prime} \in S_{q} F\left(v^{\prime}\right)$. Furthermore,

$$
\theta^{\prime}:=\varangle_{q}\left(w^{\prime}, v^{\prime}\right)=\varangle_{p}(w, v)=: \theta,
$$

where $\theta, \theta^{\prime} \in[-\pi, \pi]$. To see this we note that $c_{v}$ and $c_{w}$ respectively $c_{v^{\prime}}$ and $c_{w^{\prime}}$ are lying in the Euclidean spaces $F(v)$ resp. $F\left(v^{\prime}\right)$. Therefore, we have

$$
d\left(c_{v}(t), c_{w}(t)\right)=2 t \sin \left(\frac{\theta}{2}\right) \quad \text { and } \quad d\left(c_{v^{\prime}}(t), c_{w^{\prime}}(t)\right)=2 t \sin \left(\frac{\theta^{\prime}}{2}\right)
$$

and the triangle inequality implies

$$
\begin{aligned}
2 t\left|\sin \left(\frac{\theta}{2}\right)-\sin \left(\frac{\theta^{\prime}}{2}\right)\right| & =\left|d\left(c_{v}(t), c_{w}(t)\right)-d\left(c_{v^{\prime}}(t), c_{w^{\prime}}(t)\right)\right| \\
& \leq\left|d\left(c_{v}(t), c_{v^{\prime}}(t)\right)+d\left(c_{w^{\prime}}(t), c_{w}(t)\right)\right| \leq A
\end{aligned}
$$

for some constant $A>0$ and for all $t \geq 0$. Hence $\theta=\theta^{\prime}$. In particular, for $w=-v$ we obtain

$$
w^{\prime}=(-v)^{\prime}=-\left(v^{\prime}\right)
$$

since

$$
\varangle_{q}\left((-v)^{\prime}, v^{\prime}\right)=\varangle_{p}(-v, v)=\pi
$$

Consequently

$$
d\left(c_{v^{\prime}}(-t), c_{v}(-t)\right)=d\left(c_{-\left(v^{\prime}\right)}(t), c_{-v}(t)\right)=d\left(c_{(-v)^{\prime}}(t), c_{-v}(t)\right) \leq d(p, q)
$$

for all $t \geq 0$. Since $d\left(c_{v}(t), c_{v^{\prime}}(t)\right)$ converges to 0 for $t \rightarrow \infty$ we have $v=v^{\prime}$ which is in contradiction to the assumption. Hence the rank of $X$ must be one. 


\section{Appendix}

In this appendix we collect properties of $(1,1)$-tensors which are used in this paper.

Definition 7.1. Let $B: I \rightarrow$ End $N(c)$ be a $(1,1)$-tensor along a geodesic $c: I \rightarrow$ $M$. Then for $t_{0}, t \in I$ we define $\int_{t_{0}}^{t} B(s) d s: I \rightarrow$ End $N(c)$ via

$$
\left\langle\int_{t_{0}}^{t} B(s) d s x_{t}, y_{t}\right\rangle:=\int_{t_{0}}^{t}\left\langle B(s) x_{s}, y_{s}\right\rangle d s,
$$

where $x_{t}, y_{t}$ are orthogonal parallel vector fields along $c$.

Remark. If $B: I \rightarrow$ End $N(c)$ is a symmetric, positive definite $(1,1)$-tensor along a geodesic $c: I \rightarrow M$, then $\int_{t_{0}}^{t} B(s) d s: I \rightarrow$ End $N(c)$ is symmetric and positive definite as well. Furthermore, the following estimates hold:

$$
\begin{aligned}
\left\|\int_{t_{0}}^{t} B(s) d s\right\| & \leq \int_{t_{0}}^{t}\|B(s)\| d s, \\
\left\|\left(\int_{t_{0}}^{t} B(s) d s\right)^{-1}\right\|^{-1} & \geq \int_{t_{0}}^{t}\left\|B(s)^{-1}\right\|^{-1} d s .
\end{aligned}
$$

Proposition 7.2. Let $M$ be a Riemannian manifold. Let $Y: I \rightarrow$ End $N(c)$ be a Lagrange tensor along a geodesic $c: I \rightarrow M$ which is nonsingular for all $t \in I$. Then for $t_{0} \in I$ and any other Jacobi tensor $Z$ along $c$, there exist constant tensors $C_{1}$ and $C_{2}$ such that

$$
Z(t)=Y(t)\left(\int_{t_{0}}^{t}\left(Y^{*} Y\right)^{-1}(s) d s C_{1}+C_{2}\right)
$$

for all $t \in I$.

Proof. Since $Y$ is nonsingular there exists a $(1,1)$-tensor $B$ along $C$ such that

$$
Z(t)=Y(t) B(t) .
$$

Differentiating this equation twice yields

$$
Z^{\prime \prime}(t)=Y^{\prime \prime}(t) B(t)+2 Y^{\prime}(t) B^{\prime}(t)+Y(t) B^{\prime \prime}(t) .
$$

Since $Y$ and $Z$ are Jacobi tensors, we obtain

$$
Z^{\prime \prime}(t)=-R(t) Z(t)=-R(t) Y(t) B(t) \quad \text { and } \quad Y^{\prime \prime}(t)=-R(t) Y(t)
$$


and, therefore,

$$
2 Y^{\prime}(t) B^{\prime}(t)+Y(t) B^{\prime \prime}(t)=0 .
$$

Since $Y$ is nonsingular $B^{\prime}$ is a solution of the differential equation

$$
2 Y^{-1}(t) Y^{\prime}(t) G(t)+G^{\prime}(t)=0 .
$$

Since $Y$ is a Lagrange tensor we have

$$
W(Y, Y)=Y^{*^{\prime}}(t) Y(t)-Y^{*}(t) Y^{\prime}(t)=0 .
$$

Therefore, $G(t):=\left(Y^{*} Y\right)^{-1}(t)$ is a solution of (7.3) as the following computation shows:

$$
\begin{aligned}
G^{\prime}(t) & =\left(\left(Y^{*} Y\right)^{-1}\right)^{\prime}(t)=-\left(Y^{*} Y\right)^{-1}(t)\left(Y^{*} Y\right)^{\prime}(t)\left(Y^{*} Y\right)^{-1}(t) \\
& =-Y^{-1}(t) Y^{*^{-1}}(t)\left(Y^{*^{\prime}}(t) Y(t)+Y^{*}(t) Y^{\prime}(t)\right) Y^{-1}(t) Y^{*^{-1}}(t) \\
& =-2 Y^{-1}(t) Y^{*^{-1}}(t) Y^{*}(t) Y^{\prime}(t) Y^{-1}(t) Y^{*^{-1}}(t) \\
& =-2 Y^{-1}(t) Y^{\prime}(t) G(t) .
\end{aligned}
$$

Hence, an arbitrary solution of (7.3) is of the form $G(t)=\left(Y^{*} Y\right)^{-1}(t) C$, where $C$ is a constant tensor along $c$. Therefore, $B^{\prime}(t)=\left(Y^{*} Y\right)^{-1}(t) C_{1}$ and integration yields

$$
B(t)=\int_{t_{0}}^{t}\left(Y^{*} Y\right)^{-1}(s) d s C_{1}+C_{2}
$$

for constant tensors $C_{1}, C_{2}$ along $c$.

The following properties of the stable and unstable Jacobi tensors $U_{v}$ and $S_{v}$ defined in Section 2 are frequently used in this paper.

Lemma 7.3. Let $M$ be a manifold without conjugate points. Then for all $v \in S M$ we have

$$
\begin{array}{r}
S_{\phi^{u}(v)}(t)=S_{v}(t+u) S_{v}^{-1}(u) \quad \text { and } \quad U_{\phi^{u}(v)}(t)=U_{v}(t+u) U_{v}^{-1}(u), \\
S_{\phi^{t}(v)}^{\prime}(0)=S_{v}^{\prime}(t) S_{v}^{-1}(t) \quad \text { and } U_{\phi^{t}(v)}^{\prime}(0)=U_{v}^{\prime}(t) U_{v}^{-1}(t) \\
U_{\phi^{t}(v)}^{\prime}(0)-S_{\phi^{t}(v)}^{\prime}(0)=U_{v}^{*^{-1}}(t)\left(U_{v}^{\prime}(0)-S_{v}^{\prime}(0)\right) S_{v}^{-1}(t) \\
=S_{v}^{*-1}(t)\left(U_{v}^{\prime}(0)-S_{v}^{\prime}(0)\right) U_{v}^{-1}(t)
\end{array}
$$

Furthermore,

$$
U_{\phi^{t}(v)}^{\prime}(0)-S_{\phi^{t}(v)}^{\prime}(0)=S_{v}^{*-1}(t)\left(\int_{-\infty}^{t}\left(S_{v}^{*} S_{v}\right)^{-1}(u) d u\right)^{-1} S_{v}^{-1}(t) .
$$


Furthermore, for all $x \in \mathscr{L}(v)$ we have $S_{v}(t) x_{t}=U_{v}(t) x_{t} \in \mathscr{L}\left(\phi^{t} v\right)$. Moreover, $S_{v}(t) y=U_{v}(t) y \in \mathscr{L}\left(\phi^{t} v\right)$ for all $y \in \mathscr{L}\left(\phi^{t} v\right)$.

Proof. Let $S_{v, r}(t)$ the Jacobi tensor along the geodesic $c_{v}(t)$ with $S_{v, r}(0)=$ id and $S_{v, r}(r)=0$. Then

$$
S_{\phi_{u} v, r}(t)=S_{v, r+u}(t+u) S_{v, r+u}^{-1}(u),
$$

since both sides define for fixed $u$ and $r$ Jacobi tensors, which agree at $t=0$ and $t=r$. Taking the limit $r \rightarrow \infty$ yields the first assertion of (7.4). The second follows with a similar argument. Differentiating this relations yields (7.5).

To prove (7.6) consider the Wronskian $W\left(U_{v}, S_{v}\right)(t)$ given by

$$
W\left(U_{v}, S_{v}\right)(t)=U_{v}^{*^{\prime}}(t) S_{v}(t)-U_{v}^{*}(t) S_{v}^{\prime}(t)=W\left(U_{v}, S_{v}\right)(0)=B_{v},
$$

where

$$
B_{v}=U_{v}^{\prime}(0)-S_{v}^{\prime}(0)
$$

This yields

$$
\left(U_{v}^{\prime}(t) U_{v}^{-1}(t)\right)^{*}-S_{v}^{\prime}(t) S_{v}^{-1}(t)=U_{v}^{*-1}(t) B_{v} S_{v}^{-1}(t) .
$$

Since

$$
\left(U_{v}^{\prime}(t) U_{v}^{-1}(t)\right)=U_{\phi^{t}(v)}^{\prime}(0) \quad \text { and } \quad S_{v}^{\prime}(t) S_{v}^{-1}(t)=S_{\phi^{t}(v)}^{\prime}(0)
$$

are symmetric (see Section 2), we obtain the first identity and taking the adjoint we obtain the second one.

To prove (7.7) consider $0<r, s$ and $t \in(-\infty, s)$. As we have shown in Section 2 the Jacobi tensor $S_{v, s}(t)$ is Lagrangian, we obtain using Proposition 7.2 the existence of a constant tensor $K_{s, r}$ such that

$$
U_{v, r}(t)=S_{v, s}(t) \int_{-r}^{t}\left(S_{v, s}^{*} S_{v, s}\right)^{-1}(u) d u K_{s, r} .
$$

Evaluating and differentiating this identity at $t=0$ we obtain the equation

$$
U_{v, r}(t)=S_{v, s}(t) \int_{-r}^{t}\left(S_{v, s}^{*} S_{v, s}\right)^{-1}(u) d u\left(U_{v, r}^{\prime}(0)-S_{v, s}^{\prime}(0)\right) .
$$

Furthermore, for all $t<s$ the limit

$$
\lim _{r \rightarrow \infty} \int_{-r}^{t}\left(S_{v, s}^{*} S_{v, s}\right)^{-1}(u) d u=\int_{-\infty}^{t}\left(S_{v, s}^{*} S_{v, s}\right)^{-1}(u) d u
$$


exists and is invertible. Hence,

$$
\left(\int_{-\infty}^{t}\left(S_{v, s}^{*} S_{v, s}\right)^{-1}(u) d u\right)^{-1} S_{v, s}(t)^{-1} U_{v}(t)=U_{v}^{\prime}(0)-S_{v, s}^{\prime}(0) .
$$

Passing to the limit $s \rightarrow \infty$, we obtain

$$
\left(\int_{-\infty}^{t}\left(S_{v}^{*} S_{v}\right)^{-1}(u) d u\right)^{-1} S_{v}(t)^{-1} U_{v}(t)=U_{v}^{\prime}(0)-S_{v}^{\prime}(0) .
$$

Inserting (7.8) into the second identity of (7.6), we obtain

$$
U_{\phi^{t}(v)}^{\prime}(0)-S_{\phi^{t}(v)}^{\prime}(0)=S_{v}^{*-1}(t)\left(\int_{-\infty}^{t}\left(S_{v}^{*} S_{v}\right)^{-1}(u) d u\right)^{-1} S_{v}^{-1}(t) .
$$

Finally, consider $x \in \mathscr{L}(v)$, i.e., $U_{v}^{\prime}(0) x=S_{v}^{\prime}(0) x$. Then, $U_{v}(t) x_{t}=S_{v}(t) x_{t}$ and $U_{v}^{\prime}(t) x_{t}=S_{v}^{\prime}(t) x_{t}$. This implies

$$
U_{\phi^{t}(v)}^{\prime}(0) U_{v}(t) x_{t}=U_{v}^{\prime}(t) U_{v}^{-1}(t) U_{v}(t) x_{t}=S_{v}^{\prime}(t) x_{t}=S_{\phi^{t}(v)}^{\prime}(0) S_{v}(t) x_{t}
$$

and, therefore, $U_{v}(t) x_{t}=S_{v}(t) x_{t} \in \mathscr{L}\left(\phi^{t}(v)\right)$.

Acknowledgement. I would like to thank Norbert Peyerimhoff for asking the question on the volume growth of noncompact harmonic manifolds formulated in the introduction, which initiated this work. Furthermore, I am grateful to him for some suggestions improving the presentation of this article.

\section{References}

[BBE] W. Ballmann, M. Brin and P. Eberlein, Structure of manifolds of nonpositive curvature I. Ann. of Math. 122 (1985), no. 2, 171-203. Zbl 0589.53047 MR 0799256

[BCG] G. Besson, G. Courtois and S. Gallot, Entropies et rigidités des espaces localement symétriques de courbure strictement négative. Geom. Funct. Anal. 5 (1995), no. 5, 731-799. Zbl 0851.53032 MR 1354289

[BFL] Y. Benoist, P. Foulon and F. Labourie, Flots d' Anosov à distributions stable et instable differéntiables. J. Amer. Math. Soc. 5 (1992), no. 1, 33-74. Zbl 0759.58035 MR 1124979

[Be] A. L. Besse, Manifolds all of whose geodesics are closed. Ergeb. Math. Grenzgeb. 93, Springer-Verlag, Berlin 1978. Zbl 0387.53010 MR 0496885

[BH] M. R. Bridson and A. Haefliger, Metric spaces of nonpositive curvature. Grundlehren Math. Wiss. 319, Springer-Verlag, Berlin 1999. Zbl 0988.53001 MR 1744486 
[Bo] J. Bolton, Conditions under which a geodesic flow is Anosov. Math. Ann. 240 (1979), no. 2, 103-113. Zbl 0382.58017 MR 0524660

[Bu] K. Burns, The flat strip theorem fails for surfaces with no conjugate points. Proc. Amer. Math. Soc. 115 (1992), no. 1, 199-206. Zbl 0759.53031 MR 1093593

[DR] E. Damek and F. Ricci, A class of nonsymmetric harmonic Riemannian spaces. Bull. Amer. Math. Soc. (N.S.) 27 (1992), no. 1, 139-142. Zbl 0755.53032 MR 1142682

[Eb] P. Eberlein, When is a geodesic flow of Anosov type? I. J. Differential Geom. 8 (1973), 437-463. Zbl 0285.58008 MR 0380891

[EO] P. Eberlein and B. O'Neill, Visibility manifolds. Pacific J. Math. 46 (1973), 45-109. Zbl 0264.53026 MR 0336648

[Es] J. H. Eschenburg, Horospheres and the stable part of the geodesic flow. Math. Z. 153 (1977), no. 3, 237-251. Zbl 0332.53028 MR 0440605

[FM] A. Freiré and R. Mañé, On the entropy of the geodesic flow in manifolds without conjugate points. Invent. Math. 69 (1982), no. 3, 375-392. Zbl 0476.58019 MR 0679763

[FL] P. Foulon and F. Labourie, Sur les variétés compactes asymptotiquement harmoniques. Invent. Math. 109 (1992), no. 1, 97-111. Zbl 0767.53030 MR 1168367

[Gr] M. Gromov, Hyperbolic groups. In Essays in group theory, Math. Sci. Res. Inst. Publ. 8, Springer-Verlag, New York 1987, 75-263. Zbl 0634.20015 MR 0919829

[Gre] L. Green, A theorem of E. Hopf. Michigan Math. J. 5 (1958), no. 1, 31-34. Zbl 0134.39601 MR 0097833

[He] J. Heber, On harmonic and asymptotically harmonic homogeneous spaces. Geom. Funct. Anal. 16 ( (2006), no. 4, 869-890. Zbl 1108.53022 MR 2255384

[K1] W. Klingenberg, Riemannian manifolds with geodesic flow of Anosov type. Ann. of Math. 99 (1974), no. 2, 1-13. Zbl 0272.53025 MR 0377980

[Kn2] G. Knieper, Mannigfaltigkeiten ohne konjugierte Punkte. Bonner Math. Schriften 168 (1986). Zbl 0601.53039 MR 0851010

[Kn1] G. Knieper, On the asymptotic geometry of nonpositively curved manifolds. Geom. Funct. Anal. 7 (1997), no. 4, 755-782. Zbl 0896.53033 MR 1465601

[Kn] G. Knieper, Hyperbolic dynamics and Riemannian geometry. In Handbook of dynamical systems, Vol. 1A, B. Hasselblatt and A. Katok, eds., North-Holland, Amsterdam 2002, 453-545. Zbl 1049.37020 MR 1928523

[Li] A. Lichnerowicz, Sur les espaces riemanniens complètement harmoniques. Bull. Soc. Math. France 72 (1944), 146-168. Zbl 0060.38506 MR 0012886

[Ma] R. Mané, On a theorem of Klingenberg. In Dynamical systems and bifurcation theory (Rio de Janeiro, 1985), M. Camacho, M. Pacifico and F. Takens, eds., Pitman Res. Notes Math. Ser. 160, Longman Scientific \& Technical, Harlow 1987, 319-345. Zbl 0633.58022 MR 0907897

[Ni] Y. Nikolayevsky, Two theorems on harmonic manifolds. Comment. Math. Helv. 80 (2005), no. 1, 29-50. Zbl 1078.53032 MR 2130564

[RSh1] A. Ranjan and H. Shah, Convexity of spheres in a manifold without conjugate points. Proc. Indian Acad. Sci. Math. Sci. 112 (2002), no. 4, 595-599. Zbl 1020.53035 MR 1941895 
[RSh2] A. Ranjan and H. Shah, Harmonic manifolds with minimal horospheres. J. Geom. Anal. 12 (2002), no. 4, 683-694. Zbl 1066.53086 MR 1916864

[RSh3] A. Ranjan and H. Shah, Busemann functions in a harmonic manifold. Geom. Dedicata 101 (2003), 167-183. Zbl 1045.53023 MR 2017901

[SW] N. E. Steenrod and J. H. C. Whitehead, Vector fields on the $n$-sphere. Proc. Nat. Acad. Sci. U.S.A. 37 (1951), 58-63. Zbl 0054.07104 MR 0041436

[Sz] Z. I. Szabó, The Lichnerowicz Conjecture on harmonic manifolds. J. Differential Geom. 31 (1990), no. 1, 1-28. Zbl 0686.53042 MR 1030663

Received September 9, 2009

Gerhard Knieper, Fakultät für Mathematik, Ruhr-Universität Bochum, 44780 Bochum, Germany

E-mail: gerhard.knieper@rub.de 